\title{
The Complexity of Computing Minimal Unidirectional Covering Sets*
}

\author{
Dorothea Baumeister \\ Institut für Informatik \\ Heinrich-Heine-Universität Düsseldorf \\ 40225 Düsseldorf, Germany \\ Felix Fischer \\ Institut für Informatik \\ Ludwig-Maximilians-Universität München \\ 80538 München, Germany
}

\author{
Felix Brandt \\ Institut für Informatik \\ Ludwig-Maximilians-Universität München \\ 80538 München, Germany \\ Jan Hoffmann \\ Institut für Informatik \\ Ludwig-Maximilians-Universität München \\ 80538 München, Germany
}

\author{
Jörg Rothe \\ Institut für Informatik \\ Heinrich-Heine-Universität Düsseldorf \\ 40225 Düsseldorf, Germany
}

July 15, 2009

\begin{abstract}
Given a binary dominance relation on a set of alternatives, a common thread in the social sciences is to identify subsets of alternatives that satisfy certain notions of stability. Examples can be found in areas as diverse as voting theory, game theory, and argumentation theory. Brandt and Fischer [BF08] proved that it is NP-hard to decide whether an alternative is contained in some inclusion-minimal unidirectional (i.e., either upward or downward) covering set. For both problems, we raise this lower bound to the $\Theta_{2}^{p}$ level of the polynomial hierarchy and provide a $\Sigma_{2}^{p}$ upper bound. Relatedly, we show that a variety of other natural problems regarding minimal or minimum-size unidirectional covering sets are hard or complete for either of NP, coNP, and $\Theta_{2}^{p}$. An important consequence of our results is that neither minimal upward nor minimal downward covering sets (even when guaranteed to exist) can be computed in polynomial time unless $\mathrm{P}=\mathrm{NP}$. This sharply contrasts with Brandt and Fischer's result that minimal bidirectional covering sets (i.e., sets that are both minimal upward and minimal downward covering sets) are polynomial-time computable.
\end{abstract}

\section{Introduction}

A common thread in the social sciences is to identify sets of alternatives that satisfy certain notions of stability according to some binary dominance relation. Applications range from cooperative to non-cooperative game theory, from social choice theory to argumentation theory, and from multi-criteria decision analysis to sports tournaments (see, e.g., [Las97, BF08] and the references therein).

*This work was supported in part by DFG grants BR-2312/6-1, RO-1202/12-1 (within the European Science Foundation's EUROCORES program LogICCC), BR 2312/3-2, and RO-1202/11-1, and by the Alexander von Humboldt Foundation's TransCoop program. This work was done in part while the fifth author was visiting the University of Rochester. 
In social choice settings, the most common dominance relation is the pairwise majority relation, where an alternative $x$ is said to dominate another alternative $y$ if the number of individuals preferring $x$ to $y$ exceeds the number of individuals preferring $y$ to $x$. McGarvey [McG53] proved that every asymmetric dominance relation can be realized via a particular preference profile, even if the individual preferences are linear. For example, Condorcet's well-known paradox says that the majority relation may contain cycles and thus does not always have maximal elements, even if all of the underlying individual preferences do. This means that the concept of maximality is rendered useless in many cases, which is why various so-called solution concepts have been proposed. Solution concepts can be used in place of maximality for nontransitive relations (see, e.g., [Las97]). In particular, concepts based on so-called covering relations - transitive subrelations of the dominance relation at hand-have turned out to be very attractive [Fis77, Mil80, Dut88].

Computational social choice is an emerging new field at the interface of social choice theory, economics, and computer science that focuses on the computational properties of social-choice-related concepts and problems [CELM07]. For example, voting procedures - and dominance-based solution concepts are closely related to the winner-determination problem in certain voting systems - have applications in artificial intelligence (especially in multiagent systems), in aggregating the web-page rankings from multiple search engines (see Dwork et al. [DKNS01]), and other domains of computer science. That is why the computational properties of voting and other social-choice-related notions have been studied in-depth recently (see the survey [FHHR09]).

This paper studies the computational complexity of problems related to the notions of upward and downward covering sets in dominance graphs. An alternative $x$ is said to upward cover another alternative $y$ if $x$ dominates $y$ and every alternative dominating $x$ also dominates $y$. The intuition is that $x$ "strongly" dominates $y$ in the sense that there is no alternative that dominates $x$ but not $y$. Similarly, an alternative $x$ is said to downward cover another alternative $y$ if $x$ dominates $y$ and every alternative dominated by $y$ is also dominated by $x$. The intuition here is that $x$ "strongly" dominates $y$ in the sense that there is no alternative dominated by $y$ but not by $x$. A minimal upward or minimal downward covering set is defined as an inclusion-minimal set of alternatives that satisfies certain notions of internal and external stability with respect to the upward or downward covering relation [Dut88, BF08].

Recent work in theoretical computer science has addressed the computational complexity of most solution concepts proposed in the context of binary dominance (see, e.g., [Woe03,Alo06, Con06, BFH07,BF08, BFHM08]). In particular, Brandt and Fischer [BF08] have shown NP-hardness of both the problem of deciding whether an alternative is contained in some minimal upward and the problem of deciding whether an alternative is contained in some minimal downward covering set, is NP-hard. For both problems, we improve on these results by raising their NP-hardness lower bounds to the $\Theta_{2}^{p}$ level of the polynomial hierarchy, and we provide an upper bound of $\Sigma_{2}^{p}$. Moreover, we will analyze the complexity of a variety of other problems associated with minimal and minimum-size upward and downward covering sets that have not been studied before. In particular, we provide hardness and completeness results for the complexity classes NP, coNP, and $\Theta_{2}^{p}$. Remarkably, these new results imply that neither minimal upward covering sets nor minimal downward covering sets (even when guaranteed to exist) can be found in polynomial time unless $\mathrm{P}=\mathrm{NP}$. This sharply contrasts with Brandt and Fischer's result that minimal bidirectional covering sets (i.e., sets that are both minimal upward and minimal downward covering sets) are polynomial-time computable [BF08]. Note that, notwithstanding the hardness of computing minimal upward covering sets, the decision version of this search problem is trivially in P: Every dominance graph always contains a minimal upward covering set.

Our $\Theta_{2}^{p}$-hardness results apply Wagner's method [Wag87] that was useful also in other contexts (see, e.g., [Wag87,HHR97a, HR98, HW02, HRS06]). To the best of our knowledge, our constructions for the first time apply his method to problems defined in terms of minimality rather than minimum size of a solution. 


\section{Definitions and Notation}

In this section, we define the required notions and notation from social choice theory and complexity theory.

Definition 2.1 (Covering Relations) Let $A$ be a finite set of alternatives, let $B \subseteq A$, and let $\succ \subseteq A \times A$ be a dominance relation on $A$, i.e., $\succ$ is asymmetric and irreflexive 1 A dominance relation $\succ$ on a set $A$ of alternatives can be conveniently represented as a dominance graph, denoted by $(A, \succ)$, whose vertices are the alternatives from $A$, and for each $x, y \in A$ there is a directed edge from $x$ to $y$ if and only if $x \succ y$.

For any two alternatives $x$ and $y$ in $B$, define the following covering relations (see, e.g., [Fis77 Mil80. Bor837):

- $x$ upward covers $y$ in $B$, denoted by $x C_{u}^{B} y$, if $x \succ y$ and for all $z \in B, z \succ x$ implies $z \succ y$, and

- $x$ downward covers $y$ in $B$, denoted by $x C_{d}^{B} y$, if $x \succ y$ and for all $z \in B, y \succ z$ implies $x \succ z$.

When clear from the context, we omit mentioning "in B" explicitly and simply write $x C_{u} y$ rather than $x C_{u}^{B} y$, and $x C_{d} y$ rather than $x C_{d}^{B} y$.

Definition 2.2 (Uncovered Set) Let $A$ be a set of alternatives, let $B \subseteq A$ be any subset, let $\succ$ be a dominance relation on $A$, and let $C$ be a covering relation on $A$ based on $\succ$. The uncovered set of $B$ with respect to $C$ is defined as

$$
\mathrm{UC}_{C}(B)=\{x \in B \mid y C x \text { for no } y \in B\} .
$$

For notational convenience, let $\mathrm{UC}_{x}(B)=\mathrm{UC}_{C_{x}}(B)$ for $x \in\{u, d\}$, and we call $\mathrm{UC}_{u}(B)$ the upward uncovered set of $B$ and $\mathrm{UC}_{d}(B)$ the downward uncovered set of $B$.

For both the upward and the downward covering relation (henceforth unidirectional covering relations), transitivity of the relation implies nonemptiness of the corresponding uncovered set for each nonempty set of alternatives. Every upward uncovered set contains one or more minimal upward covering sets, whereas minimal downward covering sets may not always exist [BF08]. Dutta [Dut88] proposed minimal covering sets in the context of tournaments, i.e., complete dominance relations, where both notions of covering coincide. Minimal unidirectional covering sets are one of several possible generalizations to incomplete dominance relations (for more details, see [ [BF08]). The intuition underlying covering sets is that there should be no reason to restrict the selection by excluding some alternative from it (internal stability) and there should be an argument against each proposal to include an outside alternative into the selection (external stability).

Definition 2.3 (Minimal Covering Set) Let $A$ be a set of alternatives, let $\succ$ be a dominance relation on $A$, and let $C$ be a covering relation based on $\succ$. A subset $B \subseteq A$ is a covering set for $A$ under $C$ if the following two properties hold:

- Internal stability: $\mathrm{UC}_{C}(B)=B$.

- External stability: For all $x \in A-B, x \notin \mathrm{UC}_{C}(B \cup\{x\})$.

A covering set $M$ for $A$ under $C$ is said to be (inclusion-)minimal if no $M^{\prime} \subset M$ is a covering set for $A$ under $C$.

\footnotetext{
${ }^{1}$ In general, $\succ$ need not be transitive or complete. For alternatives $x$ and $y, x \succ y$ (equivalently, $(x, y) \in \succ$ ) is interpreted as $x$ being strictly preferred to $y$ (and we say " $x$ dominates $y$ ”), for example as the result of a strict majority of voters preferring $x$ to $y$.
} 
Occasionally, it might be helpful to specify the dominance relation explicitly to avoid ambiguity. In such cases we refer to the dominance graph used and write, e.g., " $M$ is an upward covering set for $(A, \succ)$."

In addition to the (inclusion-)minimal unidirectional covering sets considered in [BF08], we will also consider minimum-size covering sets, i.e., unidirectional covering sets of smallest cardinality. For some of the computational problems we study, different complexities can be shown for the minimal and minimum-size versions of the problem (see Theorem 3.1 and Table 1). Specifically, we will consider six types of computational problems, for both upward and downward covering sets, and for each both their "minimal" and "minimum-size" versions. We first define the six problem types for the case of minimal upward covering sets:

1. $\mathrm{MC}_{\mathrm{u}}$-SIZE: Given a set $A$ of alternatives, a dominance relation $\succ$ on $A$, and a positive integer $k$, does there exist some minimal upward covering set for $A$ containing at most $k$ alternatives?

2. $\mathrm{MC}_{\mathrm{u}}$-MEMBER: Given a set $A$ of alternatives, a dominance relation $\succ$ on $A$, and a distinguished element $d \in A$, is $d$ contained in some minimal upward covering set for $A$ ?

3. $\mathrm{MC}_{\mathrm{u}}$-Member-All: Given a set $A$ of alternatives, a dominance relation $\succ$ on $A$, and a distinguished element $d \in A$, is $d$ contained in all minimal upward covering sets for $A$ ?

4. $\mathrm{MC}_{\mathrm{u}}$-UNIQUE: Given a set $A$ of alternatives and a dominance relation $\succ$ on $A$, does there exist a unique minimal upward covering set for $A$ ?

5. $\mathrm{MC}_{\mathrm{u}}$-TEST: Given a set $A$ of alternatives, a dominance relation $\succ$ on $A$, and a subset $M \subseteq A$, is $M$ a minimal upward covering set for $A$ ?

6. $\mathrm{MC}_{\mathrm{u}}$-FInD: Given a set $A$ of alternatives and a dominance relation $\succ$ on $A$, find a minimal upward covering set for $A$.

If we replace "upward" by "downward" above, we obtain the six corresponding "downward covering" versions, denoted by $\mathrm{MC}_{\mathrm{d}}$-Size, MC $\mathrm{d}_{\mathrm{d}}$-Member, MC $\mathrm{d}_{\mathrm{d}}$-Member-All, $\mathrm{MC}_{\mathrm{d}}$-UniQue, MC $\mathrm{d}_{\mathrm{d}}$-Test, and $\mathrm{MC}_{\mathrm{d}}$-FIND. And if we replace "minimal" by "minimum-size" in the twelve problems just defined, we obtain the corresponding "minimum-size" versions: $\mathrm{MSC}_{\mathrm{u}}$-SIZE, $\mathrm{MSC}_{\mathrm{u}}$-MEMBER, $\mathrm{MSC}_{\mathrm{u}}$-MEMBER-ALL, MSC $_{\mathrm{u}}$-Unique, MSC $\mathrm{u}_{\mathrm{u}}$-Test, MSC $_{\mathrm{u}}$-FInd, MSC $\mathrm{d}_{\mathrm{d}}$-Size, MSC $\mathrm{d}_{\mathrm{d}}$-Member, MSC $\mathrm{d}_{\mathrm{d}}$-Member-All, MSC $_{\mathrm{d}}$-UNIQUE, $\mathrm{MSC}_{\mathrm{d}}$-TEST, and $\mathrm{MSC}_{\mathrm{d}}$-FIND.

Note that the four problems $\mathrm{MC}_{\mathrm{u}}$-FIND, $\mathrm{MC}_{\mathrm{d}}$-FIND, $\mathrm{MSC}_{\mathrm{u}}$-FIND, and $\mathrm{MSC}_{\mathrm{d}}$-FIND are search problems, whereas the other twenty problems are decision problems.

We assume that the reader is familiar with the basic notions of complexity theory, such as polynomial-time many-one reducibility and the related notions of hardness and completeness, and also with standard complexity classes such as P, NP, coNP, and the polynomial hierarchy [MS72] (see also, e.g., the textbooks [Pap94, Rot05]). In particular, coNP is the class of sets whose complements are in NP. $\Sigma_{2}^{p}=\mathrm{NP}^{\mathrm{NP}}$, the second level of the polynomial hierarchy, consists of all sets that can be solved by an NP oracle machine that has access (in the sense of a Turing reduction) to an NP oracle set such as SAT. SAT denotes the satisfiability problem of propositional logic, which is one of the standard NP-complete problems (see, e.g., Garey and Johnson [GJ79]) and is defined as follows: Given a boolean formula in conjunctive normal form, does there exist a truth assignment to its variables that satisfies the formula?

Papadimitriou and Zachos [PZ83] introduced the class of problems that can be decided by a P machine that accesses its NP oracle in a parallel manner. This class is also known as the $\Theta_{2}^{p}$ level of the polynomial hierarchy (see Wagner [Wag90]), and has been shown to coincide with the class of problems solvable in polynomial time via asking $\mathscr{O}(\log n)$ sequential Turing queries to NP (see [Hem87, KSW87]). Equivalently, $\Theta_{2}^{p}$ is the 


\begin{tabular}{lllll}
\hline Problem Type & \multicolumn{1}{c}{$\mathrm{MC}_{\mathrm{u}}$} & \multicolumn{1}{c}{$\mathrm{MSC}_{\mathrm{u}}$} & \multicolumn{1}{c}{$\mathrm{MC}_{\mathrm{d}}$} & MSC $_{\mathrm{d}}$ \\
\hline SIZE & NP-complete & NP-complete & NP-complete & NP-complete \\
MEMBER & $\Theta_{2}^{p}$-hard and in $\Sigma_{2}^{p}$ & $\Theta_{2}^{p}$-complete & $\Theta_{2}^{p}$-hard and in $\Sigma_{2}^{p}$ & coNP-hard and in $\Theta_{2}^{p}$ \\
MEMBER-ALL & coNP-complete $[\overline{\mathrm{BF} 08]}$ & $\Theta_{2}^{p}$-complete & coNP-complete $[\mathrm{BF08}]$ & coNP-hard and in $\Theta_{2}^{p}$ \\
UNIQUE & coNP-hard and in $\Sigma_{2}^{p}$ & coNP-hard and in $\Theta_{2}^{p}$ & coNP-hard and in $\Sigma_{2}^{p}$ & coNP-hard and in $\Theta_{2}^{p}$ \\
TEST & coNP-complete & coNP-complete & coNP-complete & coNP-complete \\
FIND & not in polynomial & not in polynomial & not in polynomial & not in polynomial \\
& time unless $\mathrm{P}=\mathrm{NP}$ & time unless $\mathrm{P}=\mathrm{NP}$ & time unless $\mathrm{P}=\mathrm{NP}$ & time unless $\mathrm{P}=\mathrm{NP}$ \\
& & & (follows from $[\mathrm{BF08}])$ & \\
\hline
\end{tabular}

Table 1: Overview of complexity results for the various types of covering set problems. As indicated, previously known results are due to Brandt and Fischer [BF08]; all other results are new to this paper.

closure of NP under polynomial-time truth-table reductions. It follows immediately from the definitions that $\mathrm{P} \subseteq \mathrm{NP} \cap \operatorname{coNP} \subseteq \mathrm{NP} \cup \operatorname{coNP} \subseteq \Theta_{2}^{p} \subseteq \Sigma_{2}^{p}$.

$\Theta_{2}^{p}$ captures the complexity of various optimization problems. For example, the problem of testing whether the size of a maximum clique in a given graph is an odd number, the problem of deciding whether two given graphs have minimum vertex covers of the same size, and the problem of recognizing those graphs for which certain heuristics yield good approximations for the size of a maximum independent set or for the size of a minimum vertex cover each are known to be complete for $\Theta_{2}^{p}$ (see [Wag87, HR98, HRS06]). Hemaspaandra and Wechsung [HW02] proved that the minimization problem for boolean formulas is $\Theta_{2}^{p}$-hard. In the field of computational social choice, the winner problems for Dodgson [Dod76], Young [You77], and Kemeny [Kem59] elections have been shown to be $\Theta_{2}^{p}$-complete in the nonunique-winner model [HHR97a, RSV03, HSV05], and also in the unique-winner model [HHR08].

\section{Results and Discussion}

Results. Brandt and Fischer [BF08] proved that it is NP-hard to decide whether a given alternative is contained in some minimal unidirectional covering set. Using the notation of this paper, their results state that the problems $\mathrm{MC}_{\mathrm{u}}$-MEMBER and $\mathrm{MC}_{\mathrm{d}}$-MEMBER are NP-hard. The question of whether these two problems are NP-complete or of higher complexity was left open in [BF08]. Our contribution is

1. to raise Brandt and Fischer's NP-hardness lower bounds for $\mathrm{MC}_{\mathrm{u}}-\mathrm{MEMBER}$ and $\mathrm{MC}_{\mathrm{d}}-\mathrm{MEMBER}$ to $\Theta_{2}^{p}$ hardness and to provide (simple) $\Sigma_{2}^{p}$ upper bounds for these problems, and

2. to extend the techniques we developed to apply also to the 22 other covering set problems defined in Section 2 in particular to the search problems.

Our results are stated in the following theorem.

Theorem 3.1 The complexity of the covering set problems defined in Section 2 is as shown in Table 1

The detailed proofs of the single results collected in Theorem 3.1 will be presented in Section 5, and the technical constructions establishing the properties that are needed for these proofs are given in Section 4 
Discussion. We consider the problems of finding minimal and minimum-size upward and downward covering sets $\left(\mathrm{MC}_{\mathrm{u}}\right.$-FIND, $\mathrm{MC}_{\mathrm{d}}$-FIND, $\mathrm{MSC}_{\mathrm{u}}$-FIND, and $\mathrm{MSC}_{\mathrm{d}}$-FIND) to be particularly important and natural.

Regarding upward covering sets, we stress that our result (see Theorem 5.7) that, assuming $\mathrm{P} \neq$ $\mathrm{NP}, \mathrm{MC}_{\mathrm{u}}$-FIND and $\mathrm{MSC}_{\mathrm{u}}$-FIND are hard to compute does not follow directly from the NP-hardness of $\mathrm{MC}_{\mathrm{u}}$-MEMBER in any obvious way 2 Our reduction that raises the lower bound of $\mathrm{MC}_{\mathrm{u}}$-MEMBER from NPhardness to $\Theta_{2}^{p}$-hardness, however, also allows us to prove that $\mathrm{MC}_{\mathrm{u}}$-FIND and $\mathrm{MSC}_{\mathrm{u}}$-FIND cannot be solved in polynomial time unless $\mathrm{P}=\mathrm{NP}$.

Regarding downward covering sets, that $\mathrm{MC}_{\mathrm{d}}$-FIND cannot be computed in polynomial time unless $\mathrm{P}=\mathrm{NP}$ is an immediate consequence of Brandt and Fischer's result that it is NP-complete to decide whether there exists a minimal downward covering set [BF08, Thm. 9]. We provide as Theorem 5.13 an alternative proof based on our reduction showing that $\mathrm{MC}_{\mathrm{d}}$-MEMBER is $\Theta_{2}^{p}$-hard. In contrast to Brandt and Fischer's proof, our proof shows the hardness of $\mathrm{MC}_{\mathrm{d}}$-FIND even when the existence of a (minimal) downward covering set is guaranteed. As indicated in Table 1, coNP-completeness of $\mathrm{MC}_{\mathrm{u}}$-MEMBER-ALL and $\mathrm{MC}_{\mathrm{d}}$-MEMBER-ALL was also shown previously by Brandt and Fischer [BF08].

As mentioned above, the two problems $\mathrm{MC}_{\mathrm{u}}$-MEMBER and $\mathrm{MC}_{\mathrm{d}}$-MEMBER were already known to be NPhard [BF08] and are here shown to be even $\Theta_{2}^{p}$-hard. One may naturally wonder whether raising their (or any problem's) lower bound from NP-hardness to $\Theta_{2}^{p}$-hardness gives us any more insight into the problem's inherent computational complexity. After all, $\mathrm{P}=\mathrm{NP}$ if and only if $\mathrm{P}=\Theta_{2}^{p}$. However, this question is a bit more subtle than that and has been discussed carefully by Hemaspaandra et al. [HHR97b]. They make the case that the answer to this question crucially depends on what one considers to be the most natural computational model. In particular, they argue that raising NP-hardness to $\Theta_{2}^{p}$-hardness potentially (i.e., unless longstanding open problems regarding the separation of the corresponding complexity classes could be solved) is an improvement in terms of randomized polynomial time and in terms of unambiguous polynomial time [HHR97b].

\section{Constructions}

In this section, we provide the constructions that will be used in Section 5 to obtain the new complexity results for the problems defined in Section 2 ,

\subsection{Minimal and Minimum-Size Upward Covering Sets}

We start by giving the constructions that will be used for establishing results on the minimal and minimum-size upward covering set problems. Brandt and Fischer [BF08] proved the following result. Since we will need their reduction in Construction 4.7 and Section 5, we give a proof sketch for Theorem 4.1.

Theorem 4.1 (Brandt and Fischer [|BF08]) Deciding whether a designated alternative is contained in some minimal upward covering set for a given dominance graph is $\mathrm{NP}$-hard. That is, $\mathrm{MC}_{\mathrm{u}}$-MEMBER is NP-hard.

\footnotetext{
${ }^{2}$ The decision version of $\mathrm{MC}_{\mathrm{u}}$-FIND is: Given a dominance graph, does it contain a minimal upward covering set? However, this question has always an affirmative answer, so the decision version of $\mathrm{MC}_{\mathrm{u}}$-FIND is trivially in P. Note also that $\mathrm{MC}_{\mathrm{u}}$-FIND can be reduced in a "disjunctive truth-table" fashion to the search version of $\mathrm{MC}_{\mathrm{u}}$-MEMBER ("Given a dominance graph $(A, \succ$ ) and an alternative $d \in A$, find some minimal upward covering set for $A$ that contains $d$ ") by asking this oracle set about all alternatives in parallel. So $\mathrm{MC}_{\mathrm{u}}$-FIND is no harder (with respect to disjunctive truth-table reductions) than that problem. The converse, however, is not at all obvious. Brandt and Fischer's results only imply the hardness of finding an alternative that is contained in all minimal upward covering sets [BF08].
} 


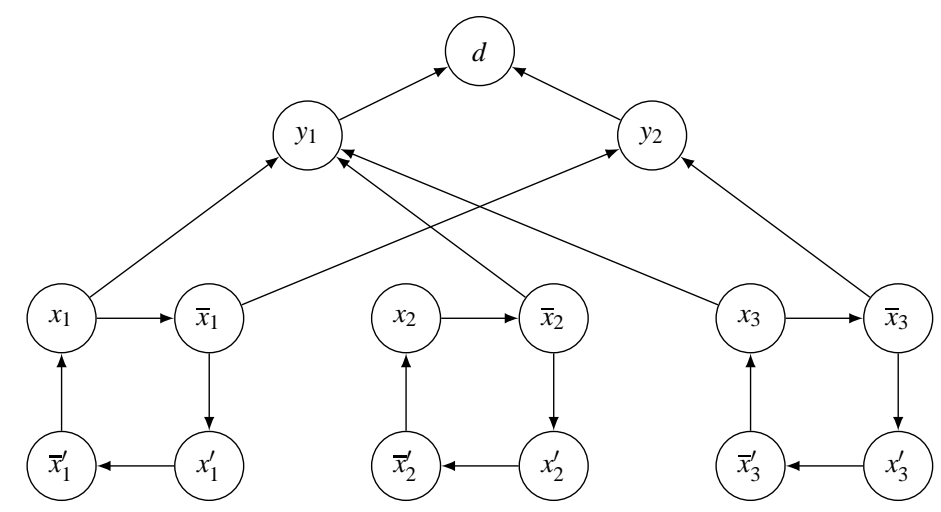

Figure 1: Dominance graph for Theorem 4.1, example for the formula $\left(v_{1} \vee \neg v_{2} \vee v_{3}\right) \wedge\left(\neg v_{1} \vee \neg v_{3}\right)$.

Proof Sketch. NP-hardness is shown by a reduction from SAT. Given a boolean formula in conjunctive normal form, $\varphi\left(v_{1}, v_{2}, \ldots, v_{n}\right)=c_{1} \wedge c_{2} \wedge \cdots \wedge c_{r}$, over the set $V=\left\{v_{1}, v_{2}, \ldots, v_{n}\right\}$ of variables, construct an instance $(A, \succ, d)$ of $\mathrm{MC}_{\mathrm{u}}$-MEMBER as follows. The set of alternatives is

$$
A=\left\{x_{i}, \bar{x}_{i}, x_{i}^{\prime}, \bar{x}_{i}^{\prime} \mid v_{i} \in V\right\} \cup\left\{y_{j} \mid c_{j} \text { is a clause in } \varphi\right\} \cup\{d\},
$$

where $d$ is the distinguished alternative whose membership in a minimal upward covering set for $A$ is to be decided, and the dominance relation $\succ$ is defined by:

- For each $i, 1 \leq i \leq n$, there is a cycle $x_{i} \succ \bar{x}_{i} \succ x_{i}^{\prime} \succ \bar{x}_{i}^{\prime} \succ x_{i}$;

- if variable $v_{i}$ occurs in clause $c_{j}$ as a positive literal, then $x_{i} \succ y_{j}$;

- if variable $v_{i}$ occurs in clause $c_{j}$ as a negative literal, then $\bar{x}_{i} \succ y_{j}$; and

- for each $j, 1 \leq j \leq r$, we have $y_{j} \succ d$.

As an example of this reduction, Figure 1 shows the dominance graph resulting from the formula $\left(v_{1} \vee\right.$ $\left.\neg v_{2} \vee v_{3}\right) \wedge\left(\neg v_{1} \vee \neg v_{3}\right)$, which is satisfiable, for example via the truth assignment that sets each of $v_{1}, v_{2}$, and $v_{3}$ to false. Note that in this case the set $\left\{\bar{x}_{1}, \bar{x}_{1}^{\prime}, \bar{x}_{2}, \bar{x}_{2}^{\prime}, \bar{x}_{3}, \bar{x}_{3}^{\prime}\right\} \cup\{d\}$ is a minimal upward covering set for $A$, so there indeed exists a minimal upward covering set for $A$ that contains the designated alternative $d$. In general, Brandt and Fischer [BF08] proved that there exists a satisfying assignment for $\varphi$ if and only if $d$ is contained in some minimal upward covering set for $A$.

As we will use this reduction to prove results for both $\mathrm{MC}_{\mathrm{u}}$-MEMBER and some of the other problems stated in Section 2, we now analyze the minimal and minimum-size upward covering sets of the dominance graph constructed in the proof sketch of Theorem 4.1, Brandt and Fischer [BF08] showed that each minimal upward covering set for $A$ contains exactly two of the four alternatives corresponding to any of the variables, i.e., either $x_{i}$ and $x_{i}^{\prime}$, or $\overline{x_{i}}$ and $\overline{x_{i}}, 1 \leq i \leq n$. We now assume that if $\varphi$ is not satisfiable then for each truth assignment to the variables of $\varphi$, at least two clauses are unsatisfied (which can be ensured, if needed, by adding two dummy variables). It follows that every minimal upward covering set for $A$ not containing alternative $d$ must consist of at least $2 n+2$ alternatives, and every minimal upward covering set for $A$ containing $d$ consists of exactly $2 n+1$ alternatives. Thus, $\varphi$ is satisfiable if and only if every minimum-size upward covering set consists of $2 n+1$ alternatives and contains $d$. 
We now provide another construction that transforms a given boolean formula into a dominance graph with quite different properties.

Construction 4.2 (To be used for showing coNP-hardness for upward covering set problems) Given a boolean formula in conjunctive normal form, $\varphi\left(w_{1}, w_{2}, \ldots, w_{k}\right)=f_{1} \wedge f_{2} \wedge \cdots \wedge f_{\ell}$, over the set $W=$ $\left\{w_{1}, w_{2}, \ldots, w_{k}\right\}$ of variables, we construct a set of alternatives $A$ and a dominance relation $\succ$ on $A$.

The set of alternatives is $A=\left\{u_{i}, \bar{u}_{i}, u_{i}^{\prime}, \bar{u}_{i}^{\prime} \mid w_{i} \in W\right\} \cup\left\{e_{j}, e_{j}^{\prime} \mid f_{j}\right.$ is a clause in $\left.\varphi\right\} \cup\left\{a_{1}, a_{2}, a_{3}\right\}$, and the dominance relation $\succ$ is defined by:

- For each $i, 1 \leq i \leq k$, there is a cycle $u_{i} \succ \bar{u}_{i} \succ u_{i}^{\prime} \succ \bar{u}_{i}^{\prime} \succ u_{i}$;

- if variable $w_{i}$ occurs in clause $f_{j}$ as a positive literal, then $u_{i} \succ e_{j}, u_{i} \succ e_{j}^{\prime}, e_{j} \succ \bar{u}_{i}$, and $e_{j}^{\prime} \succ \bar{u}_{i}$;

- if variable $w_{i}$ occurs in clause $f_{j}$ as a negative literal, then $\bar{u}_{i} \succ e_{j}, \bar{u}_{i} \succ e_{j}^{\prime}, e_{j} \succ u_{i}$, and $e_{j}^{\prime} \succ u_{i}$;

- if variable $w_{i}$ does not occur in clause $f_{j}$, then $e_{j} \succ u_{i}^{\prime}$ and $e_{j}^{\prime} \succ \bar{u}_{i}^{\prime}$;

- for each $j, 1 \leq j \leq \ell$, we have $a_{1} \succ e_{j}$ and $a_{1} \succ e_{j}^{\prime}$; and

- there is a cycle $a_{1} \succ a_{2} \succ a_{3} \succ a_{1}$.

Figure 2 shows some parts of the dominance graph that results from the given boolean formula $\varphi$. In particular, Figure 2(a) shows that part of this graph that corresponds to some variable $w_{i}$ occuring in clause $f_{j}$ as a positive literal; Figure 2(b) shows that part of this graph that corresponds to some variable $w_{i}$ occuring in clause $f_{j}$ as a negative literal; and Figure 2(c) shows that part of this graph that corresponds to some variable $w_{i}$ not occuring in clause $f_{j}$.

As a more complete example, Figure 3 shows the entire dominance graph that corresponds to the concrete formula $\left(\neg w_{1} \vee w_{2}\right) \wedge\left(w_{1} \vee \neg w_{3}\right)$, which can be satisfied by setting, for example, each of $w_{1}, w_{2}$, and $w_{3}$ to true. A minimal upward covering set for $A$ corresponding to this assignment is $M=\left\{u_{1}, u_{1}^{\prime}, u_{2}, u_{2}^{\prime}, u_{3}, u_{3}^{\prime}, a_{1}, a_{2}, a_{3}\right\}$. Note that neither $e_{1}$ nor $e_{2}$ occurs in $M$, and none of them occurs in any other minimal upward covering set for $A$ either. For alternative $e_{1}$ this can be seen as follows for the example shown in Figure 3 . If there were a minimal upward covering set $M^{\prime}$ for $A$ containing $e_{1}$ (and thus also $e_{1}^{\prime}$, since they both are dominated by the same alternatives) then neither $\bar{u}_{1}$ nor $u_{2}$ (which dominate $e_{1}$ ) must upward cover $e_{1}$ in $M^{\prime}$, so all alternatives corresponding to the variables $w_{1}$ and $w_{2}$ (i.e., $\left\{u_{i}, \bar{u}_{i}, u_{i}^{\prime}, \bar{u}_{i}^{\prime} \mid i \in\{1,2\}\right\}$ ) would also have to be contained in $M^{\prime}$. Due to $e_{1} \succ u_{3}^{\prime}$ and $e_{1}^{\prime} \succ \bar{u}_{3}^{\prime}$, all alternatives correponding to $w_{3}$ (i.e., $\left\{u_{3}, \bar{u}_{3}, u_{3}^{\prime}, \bar{u}_{3}^{\prime}\right\}$ ) are in $M^{\prime}$ as well. Consequently, $e_{2}$ and $e_{2}^{\prime}$ are no longer upward covered and must also be in $M^{\prime}$. The alternatives $a_{1}, a_{2}$, and $a_{3}$ are contained in every minimal upward covering set for $A$. But then $M^{\prime}$ is not minimal because the upward covering set $M$, which corresponds to the satisfying assignment stated above, is a strict subset of $M^{\prime}$. Hence, $e_{1}$ cannot be contained in any minimal upward covering set for $A$.

We now show some properties of the dominance graph created by Construction 4.2 in general. We will need these properties for the proofs in Section 5. The first property, stated in Claim 4.3 , has already been seen in the example above.

Claim 4.3 Consider the dominance graph $(A, \succ)$ created by Construction 4.2 and fix any $j, 1 \leq j \leq \ell$. For each minimal upward covering set $M$ for $A$, if $M$ contains the alternative $e_{j}$ then all other alternatives are contained in $M$ as well (i.e., $A=M$ ). 


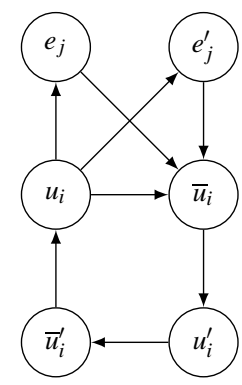

(a) $w_{i}$ occurs in $f_{j}$ as a positive literal

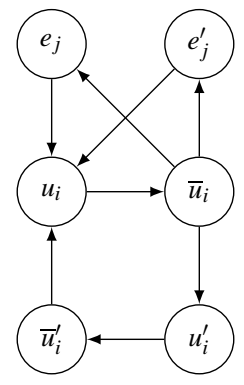

(b) $w_{i}$ occurs in $f_{j}$ as a negative literal

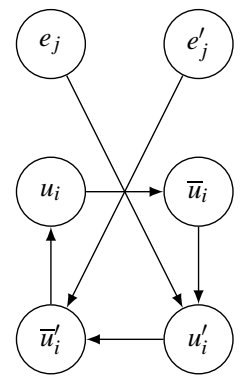

(c) $w_{i}$ does not occur in $f_{j}$

Figure 2: Parts of the dominance graph defined in Construction 4.2

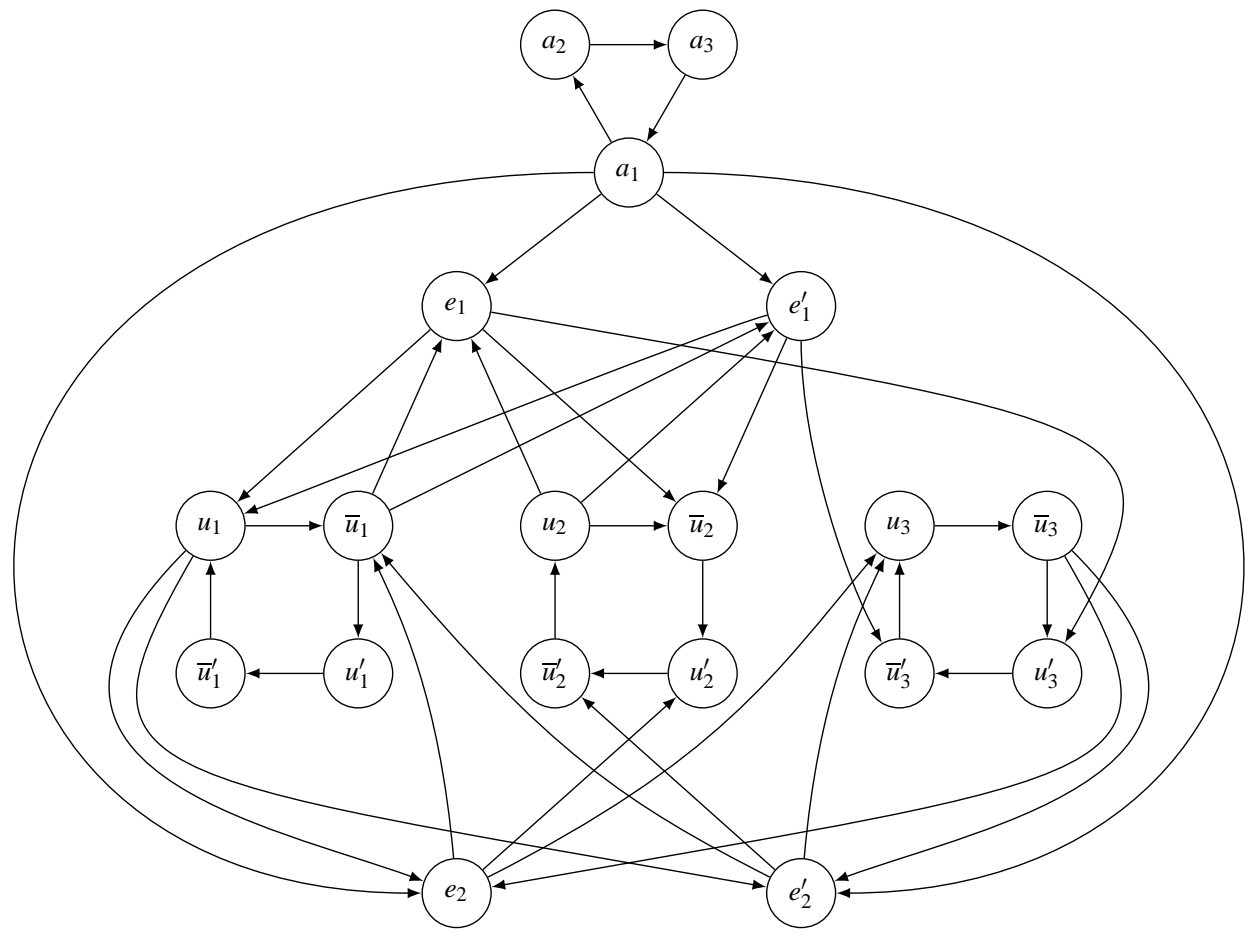

Figure 3: Dominance graph from Construction 4.2 example for the formula $\left(\neg w_{1} \vee w_{2}\right) \wedge\left(w_{1} \vee \neg w_{3}\right)$.

Proof. To simplify notation, we will prove the claim only for the case of $j=1$. However, since there is nothing special about $e_{1}$ in our argument, the same property can be shown by an analogous argument for each $j, 1 \leq j \leq \ell$.

Let $M$ be any minimal upward covering set for $A$, and suppose that $e_{1} \in M$. First note that the dominators of $e_{1}$ and $e_{1}^{\prime}$ are always the same (albeit $e_{1}$ and $e_{1}^{\prime}$ may dominate different alternatives). Thus, for each minimal upward covering set, either both $e_{1}$ and $e_{1}^{\prime}$ are contained in it, or they both are not. Thus, since $e_{1} \in M$, we have $e_{1}^{\prime} \in M$ as well.

Since the alternatives $a_{1}, a_{2}$, and $a_{3}$ form an undominated three-cycle, they each are contained in every minimal upward covering set for $A$. In particular, $\left\{a_{1}, a_{2}, a_{3}\right\} \subseteq M$. Furthermore, no alternative $e_{j}$ or $e_{j}^{\prime}$, $1 \leq j \leq \ell$, can upward cover any other alternative in $M$, because $a_{1} \in M$ and $a_{1}$ dominates $e_{j}$ and $e_{j}^{\prime}$ but none 
of the alternatives that are dominated by either $e_{j}$ or $e_{j}^{\prime}$. In particular, no alternative in any of the $k$ four-cycles $u_{i} \succ \bar{u}_{i} \succ u_{i}^{\prime} \succ \bar{u}_{i}^{\prime} \succ u_{i}$ can be upward covered by any alternative $e_{j}$ or $e_{j}^{\prime}$, and so they each must be upward covered within their cycle. For each of these cycles, every minimal upward covering set for $A$ must contain at least one of the sets $\left\{u_{i}, u_{i}^{\prime}\right\}$ and $\left\{\bar{u}_{i}, \bar{u}_{i}^{\prime}\right\}$, since at least one is needed to upward cover the other one 3

Since $e_{1} \in M$ and by internal stability, we have that no alternative from $M$ upward covers $e_{1}$. In addition to $a_{1}$, the alternatives dominating $e_{1}$ are $u_{i}$ (for each $i$ such that $w_{i}$ occurs as a positive literal in $f_{1}$ ) and $\bar{u}_{i}$ (for each $i$ such that $w_{i}$ occurs as a negative literal in $f_{1}$ ).

First assume that, for some $i, w_{i}$ occurs as a positive literal in $f_{1}$. Suppose that $\left\{u_{i}, u_{i}^{\prime}\right\} \subseteq M$. If $\bar{u}_{i}^{\prime} \notin M$ then $e_{1}$ would be upward covered by $u_{i}$, which is impossible. Thus $\bar{u}_{i}^{\prime} \in M$. But then $\bar{u}_{i} \in M$ as well, since $u_{i}$, the only alternative that could upward cover $\bar{u}_{i}$, is itself dominated by $\bar{u}_{i}^{\prime}$. For the latter argument, recall that $\bar{u}_{i}$ cannot be upward covered by any $e_{j}$ or $e_{j}^{\prime}$. Thus, we have shown that $\left\{u_{i}, u_{i}^{\prime}\right\} \subseteq M$ implies $\left\{\bar{u}_{i}, \bar{u}_{i}^{\prime}\right\} \subseteq M$. Conversely, suppose that $\left\{\bar{u}_{i}, \bar{u}_{i}^{\prime}\right\} \subseteq M$. Then $u_{i}^{\prime}$ is no longer upward covered by $\bar{u}_{i}$ and hence must be in $M$ as well. The same holds for the alternative $u_{i}$, so $\left\{\bar{u}_{i}, \bar{u}_{i}^{\prime}\right\} \subseteq M$ implies $\left\{u_{i}, u_{i}^{\prime}\right\} \subseteq M$. Summing up, if $e_{1} \in M$ then $\left\{u_{i}, u_{i}^{\prime}, \bar{u}_{i}, \bar{u}_{i}^{\prime}\right\} \subseteq M$ for each $i$ such that $w_{i}$ occurs as a positive literal in $f_{1}$.

By symmetry of the construction, an analogous argument shows that if $e_{1} \in M$ then $\left\{u_{i}, u_{i}^{\prime}, \bar{u}_{i}, \bar{u}_{i}^{\prime}\right\} \subseteq M$ for each $i$ such that $w_{i}$ occurs as a negative literal in $f_{1}$.

Now, consider any $i$ such that $w_{i}$ does not occur in $f_{1}$. We have $e_{1} \succ u_{i}^{\prime}$ and $e_{1}^{\prime} \succ \bar{u}_{i}^{\prime}$. Again, none of the sets $\left\{u_{i}, u_{i}^{\prime}\right\}$ and $\left\{\bar{u}_{i}, \bar{u}_{i}^{\prime}\right\}$ alone can be contained in $M$, since otherwise either $u_{i}$ or $\bar{u}_{i}^{\prime}$ would remain upward uncovered. Thus, $e_{1} \in M$ again implies that $\left\{u_{i}, u_{i}^{\prime}, \bar{u}_{i}, \bar{u}_{i}^{\prime}\right\} \subseteq M$.

Now it is easy to see that, since $\bigcup_{1 \leq i \leq k}\left\{u_{i}, u_{i}^{\prime}, \bar{u}_{i}, \bar{u}_{i}^{\prime}\right\} \subseteq M$ and since $a_{1}$ cannot upward cover any of the $e_{j}$ and $e_{j}^{\prime}, 1 \leq j \leq \ell$, external stability of $M$ enforces that $\bigcup_{1<j \leq \ell}\left\{e_{j}, e_{j}^{\prime}\right\} \subseteq M$. Summing up, we have shown that if $e_{1}$ is contained in any minimal upward covering set $M$ for $A$, then $M=A$.

Claim 4.4 Consider Construction 4.2. The boolean formula $\varphi$ is satisfiable if and only if there is no minimal upward covering set for $A$ that contains any of the $e_{j}, 1 \leq j \leq \ell$.

Proof. It is enough to prove the claim for the case $j=1$, since the proof for the other cases is analogous.

From left to right, suppose there is a satisfying assignment $\alpha: W \rightarrow\{0,1\}$ for $\varphi$. Define the set

$$
B_{\alpha}=\left\{a_{1}, a_{2}, a_{3}\right\} \cup\left\{u_{i}, u_{i}^{\prime} \mid \alpha\left(w_{i}\right)=1\right\} \cup\left\{\bar{u}_{i}, \bar{u}_{i}^{\prime} \mid \alpha\left(w_{i}\right)=0\right\} .
$$

Since every upward covering set for $A$ must contain $\left\{a_{1}, a_{2}, a_{3}\right\}$ and at least one of the sets $\left\{u_{i}, u_{i}^{\prime}\right\}$ and $\left\{\bar{u}_{i}, \bar{u}_{i}^{\prime}\right\}$ for each $i, 1 \leq i \leq k, B_{\alpha}$ is a (minimal) upward covering set for $A$. Let $M$ be an arbitrary minimal upward covering set for $A$. By Claim 4.3, if $e_{1}$ were contained in $M$, we would have $M=A$. But since $B_{\alpha} \subset A=M$, this contradicts the minimality of $M$. Thus $e_{1} \notin M$. (Note that every minimal upward covering set for $A$ obtained from any satisfying assignment for $\varphi$ contains exactly $2 k+3$ alternatives, and there is no minimal upward covering set of smaller size for $A$ when $\varphi$ is satisfiable. This observation will be used later on.)

From right to left, let $M$ be an arbitrary minimal upward covering set for $A$ and suppose $e_{1} \notin M$. By Claim 4.3 if any of the $e_{j}, 1<j \leq \ell$, were contained in $M$, it would follow that $e_{1} \in M$, a contradiction. Thus, $\left\{e_{j} \mid 1 \leq j \leq \ell\right\} \cap M=\emptyset$. It follows that each $e_{j}$ must be upward covered by some alternative in $M$. It is easy

\footnotetext{
${ }^{3}$ The argument is analogous to that for the construction of Brandt and Fischer [BF08] in their proof of Theorem 4.1 However, in contrast with their construction, which implies that either $\left\{x_{i}, x_{i}^{\prime}\right\}$ or $\left\{\bar{x}_{i}, \bar{x}_{i}^{\prime}\right\}, 1 \leq i \leq n$, but not both, must be contained in any minimal upward covering set for $A$ (see Figure 1 , our construction also allows for both $\left\{u_{i}, u_{i}^{\prime}\right\}$ and $\left\{\bar{u}_{i}, \bar{u}_{i}^{\prime}\right\}$ being contained in some minimal upward covering set for $A$. Informally stated, the reason is that, unlike the four-cycles in Figure 1 our four-cycles $u_{i} \succ \bar{u}_{i} \succ u_{i}^{\prime} \succ \bar{u}_{i}^{\prime} \succ u_{i}$ also have incoming edges.
} 
to see that for each $j, 1 \leq j \leq \ell$, and for each $i, 1 \leq i \leq k, e_{j}$ is upward covered in $M \cup\left\{e_{j}\right\} \supseteq\left\{u_{i}, u_{i}^{\prime}\right\}$ if $w_{i}$ occurs in $f_{j}$ as a positive literal, and $e_{j}$ is upward covered in $M \cup\left\{e_{j}\right\} \supseteq\left\{\bar{u}_{i}, \bar{u}_{i}^{\prime}\right\}$ if $w_{i}$ occurs in $e_{j}$ as a negative literal. It can never be the case that all four alternatives, $\left\{u_{i}, u_{i}^{\prime}, \bar{u}_{i}, \bar{u}_{i}^{\prime}\right\}$, are contained in $M$, because then either $e_{j}$ would no longer be upward covered in $M$ or the resulting set $M$ was not minimal. Now, $M$ induces a satisfying assignment for $\varphi$ by setting, for each $i, 1 \leq i \leq k, \alpha\left(w_{i}\right)=1$ if $u_{i} \in M$, and $\alpha\left(w_{i}\right)=0$ if $\bar{u}_{i} \in M$.

Claim 4.5 Consider Construction 4.2. The boolean formula $\varphi$ is not satisfiable if and only if there is a unique minimal upward covering set for A.

Proof. Without loss of generality, we may assume that if $\varphi$ is satisfiable then it has at least two satisfying assignments. This can be ensured, if needed, by adding dummy variables.

From left to right, suppose there is no satisfying assignment for $\varphi$. By Claim 4.4 there must be a minimal upward covering set for $A$ containing one of the $e_{j}, 1 \leq j \leq \ell$, and by Claim 4.3 this minimal upward covering set for $A$ must contain all alternatives. By reason of minimality, there cannot be another minimal upward covering set for $A$.

From right to left, suppose there is a unique minimal upward covering set for $A$. Due to our assumption that if $\varphi$ is satisfiable then there are at least two satisfying assignments, $\varphi$ cannot be satisfiable, since if it were, there would be two distinct minimal upward covering sets corresponding to these assignments (as argued in the proof of Claim 4.4).

Wagner provided a sufficient condition for proving $\Theta_{2}^{p}$-hardness that was useful in various other contexts (see, e.g., [Wag87,HHR97a, HR98,HW02,HRS06]) and is stated here as Lemma 4.6 .

Lemma 4.6 (Wagner [Wag87]) Let $S$ be some NP-complete problem, and let $T$ be any set. If there exists a polynomial-time computable function $f$ such that, for all $m \geq 1$ and all strings $x_{1}, x_{2}, \ldots, x_{2 m}$ satisfying that if $x_{j} \in S$ then $x_{j-1} \in S, 1<j \leq 2 m$, we have

$$
\left\|\left\{i \mid x_{i} \in S\right\}\right\| \text { is odd } \Longleftrightarrow f\left(x_{1}, x_{2}, \ldots, x_{2 m}\right) \in T,
$$

then $T$ is $\Theta_{2}^{p}$-hard.

We will apply Lemma 4.6 as well. In contrast with those previous results, however, one subtlety in our construction is due to the fact that we consider not only minimum-size but also (inclusion-)minimal covering sets. To the best of our knowledge, our constructions for the first time apply Wagner's technique [Wag87] to problems defined in terms of minimality/maximality rather than minimum/maximum size of a solution: $4^{4}$ In Construction 4.7below, we define a dominance graph based on Construction 4.2 and the construction presented in the proof sketch of Theorem 4.1 such that Lemma 4.6 can be applied to prove $\mathrm{MC}_{\mathrm{u}}$-MEMBER $\Theta_{2}^{p}$-hard (see Theorem 5.2), making use of the properties established in Claims 4.3, 4.4, and 4.5

Construction 4.7 (For applying Lemma 4.6 to upward covering set problems) $\quad$ We apply Wagner's Lemma with the NP-complete problem $S=\mathrm{SAT}$ and construct a dominance graph. Fix an arbitrary $m \geq 1$

\footnotetext{
${ }^{4}$ For example, recall Wagner's $\Theta_{2}^{p}$-completeness result for testing whether the size of a maximum clique in a given graph is an odd number [Wag87]. One key ingredient in his proof is to define an associative operation on graphs, $\bowtie$, such that for any two graphs $G$ and $H$, the size of a maximum clique in $G \bowtie H$ equals the sum of the sizes of a maximum clique in $G$ and one in $H$. This operation is quite simple: Just connect every vertex of $G$ with every vertex of $H$. In contrast, since minimality for minimal upward covering sets is defined in terms of set inclusion, it is not at all obvious how to define a similarly simple operation on dominance graphs such that the minimal upward covering sets in the given graphs are related to the minimal upward covering sets in the connected graph in a similarly useful way.
} 
and let $\varphi_{1}, \varphi_{2}, \ldots, \varphi_{2 m}$ be $2 m$ boolean formulas in conjunctive normal form such that if $\varphi_{j}$ is satisfiable then so is $\varphi_{j-1}$, for each $j, 1<j \leq 2 m$. Without loss of generality, we assume that for each $j, 1 \leq j \leq 2 m$, the first variable of $\varphi_{j}$ does not occur in all clauses of $\varphi_{j}$. Furthermore we require $\varphi_{j}$ to have at least two unsatisfied clauses if $\varphi_{j}$ is not satisfiable, and to have at least two satisfying assignments if $\varphi_{j}$ is satisfiable. It is easy to see that if $\varphi_{j}$ does not have these properties, it can be transformed into a formula that does have them, without affecting the satisfiability of the formula.

We will now define a polynomial-time computable function $f$, which maps the given $2 m$ boolean formulas to a dominance graph $(A, \succ)$ with useful properties for upward covering sets. Define $A=\bigcup_{j=1}^{2 m} A_{j}$ and the dominance relation $\succ$ on $A$ by

$$
\left(\bigcup_{j=1}^{2 m} \succ_{j}\right) \cup\left(\bigcup_{i=1}^{m}\left\{\left(u_{1,2 i}^{\prime}, d_{2 i-1}\right),\left(\bar{u}_{1,2 i}^{\prime}, d_{2 i-1}\right)\right\}\right) \cup\left(\bigcup_{i=2}^{m}\left\{\left(d_{2 i-1}, z\right) \mid z \in A_{2 i-2}\right\}\right),
$$

where we use the following notation:

1. For each $i, 1 \leq i \leq m$, let $\left(A_{2 i-1}, \succ_{2 i-1}\right)$ be the dominance graph that results from the formula $\varphi_{2 i-1}$ according to Brandt and Fischer's construction given in the proof sketch of Theorem 4.1] We use the same names for the alternatives in $A_{2 i-1}$ as in that proof sketch, except that we attach the subscript $2 i-1$. For example, alternative $d$ from the proof sketch of Theorem 4.1 now becomes $d_{2 i-1}, x_{1}$ becomes $x_{1,2 i-1}, y_{1}$ becomes $y_{1,2 i-1}$, and so on.

2. For each $i, 1 \leq i \leq m$, let $\left(A_{2 i}, \succ_{2 i}\right)$ be the dominance graph that results from the formula $\varphi_{2 i}$ according to Construction 4.2 We use the same names for the alternatives in $A_{2 i}$ as in that construction, except that we attach the subscript $2 i$. For example, alternative $a_{1}$ from Construction 4.2 now becomes $a_{1,2 i}, e_{1}$ becomes $e_{1,2 i}$, $u_{1}$ becomes $u_{1,2 i}$, and so on.

3. For each $i, 1 \leq i \leq m$, connect the dominance graphs $\left(A_{2 i-1}, \succ_{2 i-1}\right)$ and $\left(A_{2 i}, \succ_{2 i}\right)$ as follows. Let $u_{1,2 i}, \bar{u}_{1,2 i}, u_{1,2 i}^{\prime}, \bar{u}_{1,2 i}^{\prime} \in A_{2 i}$ be the four alternatives in the cycle corresponding to the first variable of $\varphi_{2 i}$. Then both $u_{1,2 i}^{\prime}$ and $\bar{u}_{1,2 i}^{\prime}$ dominate $d_{2 i-1}$. The resulting dominance graph is denoted by $\left(B_{i}, \succ_{i}^{B}\right)$.

4. Connect the $m$ dominance graphs $\left(B_{i}, \succ_{i}^{B}\right), 1 \leq i \leq m$, as follows: For each $i, 2 \leq i \leq m, d_{2 i-1}$ dominates all alternatives in $A_{2 i-2}$.

The dominance graph $(A, \succ)$ is sketched in Figure 4 Clearly, $(A, \succ)$ is computable in polynomial time.

Before we use this construction to obtain $\Theta_{2}^{p}$-hardness results for some of our upward covering set problems in Section 5, we will again show some useful properties of the constructed dominance graph, and we first consider the dominance graph $\left(B_{i}, \succ_{i}^{B}\right)$ (see Step 3 in Construction 4.7) separately 5 for any fixed $i$ with $1 \leq$ $i \leq m$. Doing so will simplify our argument for the whole dominance graph $(A, \succ)$. Recall that $\left(B_{i}, \succ_{i}^{B}\right)$ results from the formulas $\varphi_{2 i-1}$ and $\varphi_{2 i}$.

Claim 4.8 Consider Construction 4.7 Alternative $d_{2 i-1}$ is contained in some minimal upward covering set for $\left(B_{i}, \succ_{i}^{B}\right)$ if and only if $\varphi_{2 i-1}$ is satisfiable and $\varphi_{2 i}$ is not satisfiable.

\footnotetext{
${ }^{5}$ Note that our argument about $\left(B_{i}, \succ_{i}^{B}\right)$ can be used to show, in effect, DP-hardness of upward covering set problems, where DP is the class of differences of any two NP sets [PY84]. Note that DP is the second level of the boolean hierarchy over NP (see Cai et al. $\left.\mathrm{CGH}^{+} 88 \mathrm{CGH}^{+} 89\right]$ ), and it holds that $\mathrm{NP} \cup \operatorname{coNP} \subseteq \mathrm{DP} \subseteq \Theta_{2}^{p}$. Wagner [Wag87] proved appropriate analogs of Lemma 4.6 for each level of the boolean hierarchy. In particular, the analogous criterion for DP-hardness is obtained by using the wording of Lemma 4.6 except with the value of $m=1$ being fixed.
} 


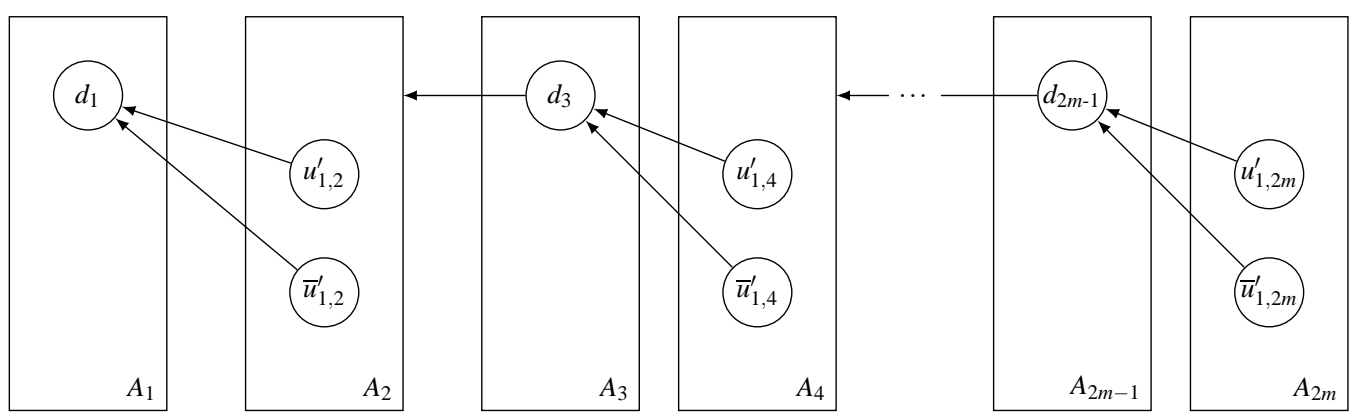

Figure 4: Dominance graph from Construction 4.7 Most alternatives, and all edges between pairs of alternatives, in $A_{j}$ for $1 \leq j \leq 2 m$ have been omitted. All edges between alternatives in $A_{i}$ and alternatives in $A_{j}$ for $i \neq j$ are shown. An edge incident to a set of alternatives represents an edge incident to each alternative in the set.

Proof. Distinguish the following three cases.

Case 1: $\varphi_{2 i-1} \in$ SAT and $\varphi_{2 i} \in$ SAT. Since $\varphi_{2 i}$ is satisfiable, it follows from the proof of Claim 4.3 that for each minimal upward covering set $M$ for $\left(B_{i}, \succ_{i}^{B}\right)$, either $\left\{u_{1,2 i}, u_{1,2 i}^{\prime}\right\} \subseteq M$ or $\left\{\bar{u}_{1,2 i}, \bar{u}_{1,2 i}^{\prime}\right\} \subseteq M$, but not both, and that none of the $e_{j, 2 i}$ and $e_{j, 2 i}^{\prime}$ is in $M$. If $\bar{u}_{1,2 i}^{\prime} \in M$ but $u_{1,2 i}^{\prime} \notin M$, then $d_{2 i-1} \notin \mathrm{UC}_{u}(M)$, since $\bar{u}_{1,2 i}^{\prime}$ upward covers $d_{2 i-1}$ within $M$. If $u_{1,2 i}^{\prime} \in M$ but $\bar{u}_{1,2 i} \notin M$, then $d_{2 i-1} \notin \mathrm{UC}_{u}(M)$, since $u_{1,2 i}^{\prime}$ upward covers $d_{2 i-1}$ within $M$. Hence, by internal stability, $d_{2 i-1}$ is not contained in $M$.

Case 2: $\varphi_{2 i-1} \notin \mathrm{SAT}$ and $\varphi_{2 i} \notin \mathrm{SAT}$. Since $\varphi_{2 i-1} \notin \mathrm{SAT}$, it follows from the proof of Theorem 4.1 that each minimal upward covering set $M$ for $\left(B_{i}, \succ_{i}^{B}\right)$ contains at least one alternative $y_{j, 2 i-1}$ (corresponding to some clause of $\varphi_{2 i-1}$ ) that upward covers $d_{2 i-1}$. Thus $d_{2 i-1}$ cannot be in $M$, again by internal stability.

Case 3: $\varphi_{2 i-1} \in \mathrm{SAT}$ and $\varphi_{2 i} \notin \mathrm{SAT}$. Since $\varphi_{2 i-1} \in \mathrm{SAT}$, it follows from the proof of Theorem 4.1 that there exists a minimal upward covering set $M^{\prime}$ for $\left(A_{2 i-1}, \succ_{2 i-1}\right)$ that corresponds to a satisfying truth assignment for $\varphi_{2 i-1}$. In particular, none of the $y_{j, 2 i-1}$ is in $M^{\prime}$. On the other hand, since $\varphi_{2 i} \notin \mathrm{SAT}$, it follows from Claim 4.5 that $A_{2 i}$ is the only minimal upward covering set for $\left(A_{2 i}, \succ_{2 i}\right)$. Define $M=M^{\prime} \cup A_{2 i}$. It is easy to see that $M$ is a minimal upward covering set for $\left(B_{i}, \succ_{i}^{B}\right)$, since the only edges between $A_{2 i-1}$ and $A_{2 i}$ are those from $\bar{u}_{1,2 i}^{\prime}$ and $u_{1,2 i}^{\prime}$ to $d_{2 i-1}$, and both $\bar{u}_{1,2 i}^{\prime}$ and $u_{1,2 i}^{\prime}$ are dominated by elements in $M$ not dominating $d_{2 i-1}$.

We now show that $d_{2 i-1} \in M$. Note that $\bar{u}_{1,2 i}^{\prime}, u_{1,2 i}^{\prime}$, and the $y_{j, 2 i-1}$ are the only alternatives in $B_{i}$ that dominate $d_{2 i-1}$. Since none of the $y_{j, 2 i-1}$ is in $M$, they do not upward cover $d_{2 i-1}$. Also, $u_{1,2 i}^{\prime}$ doesn't upward cover $d_{2 i-1}$, since $\bar{u}_{1,2 i} \in M$ and $\bar{u}_{1,2 i}$ dominates $u_{1,2 i}^{\prime}$ but not $d_{2 i-1}$. On the other hand, by our assumption that the first variable of $\varphi_{2 i}$ does not occur in all clauses, there exist alternatives $e_{j, 2 i}$ and $e_{j, 2 i}^{\prime}$ in $M$ that dominate $\bar{u}_{1,2 i}^{\prime}$ but not $d_{2 i-1}$, so $\bar{u}_{1,2 i}^{\prime}$ doesn't upward cover $d_{2 i-1}$ either. Thus $d_{2 i-1} \in M$.

Note that, by our assumption on how the formulas are ordered, the fourth case (i.e., $\varphi_{2 i-1} \notin \mathrm{SAT}$ and $\varphi_{2 i} \in \mathrm{SAT}$ ) cannot occur. Thus, the proof is complete.

Claim 4.9 Consider Construction 4.7 For each $i, 1 \leq i \leq m$, let $M_{i}$ be the minimal upward covering set for $\left(B_{i}, \succ_{i}^{B}\right)$ according to the cases in the proof of Claim 4.8 Then each of the sets $M_{i}$ must be contained in every minimal upward covering set for $(A, \succ)$. 
Proof. The minimal upward covering set $M_{m}$ for $\left(B_{m}, \succ_{m}^{B}\right)$ must be contained in every minimal upward covering set for $(A, \succ)$, since no alternative in $A-B_{m}$ dominates any alternative in $B_{m}$. On the other hand, for each $i, 1 \leq i<m$, no alternative in $B_{i}$ can be upward covered by $d_{2 i+1}$ (which is the only element in $A-B_{i}$ that dominates any of the elements of $B_{i}$ ), since $d_{2 i+1}$ is dominated within every minimal upward covering set for $B_{i+1}$ (and, in particular, within $M_{i+1}$ ). Thus, each of the sets $M_{i}, 1 \leq i \leq m$, must be contained in every minimal upward covering set for $(A, \succ)$.

Claim 4.10 Consider Construction 4.7 It holds that

$\left\|\left\{i \mid \varphi_{i} \in \mathrm{SAT}\right\}\right\|$ is odd $\Longleftrightarrow d_{1}$ is contained in some minimal upward covering set $M$ for $A$.

Proof. To show (4.2) from left to right, suppose $\left\|\left\{i \mid \varphi_{i} \in \mathrm{SAT}\right\}\right\|$ is odd. Recall that for each $j, 1<j \leq 2 \mathrm{~m}$, if $\varphi_{j}$ is satisfiable then so is $\varphi_{j-1}$. Thus, there exists some $i, 1 \leq i \leq m$, such that $\varphi_{1}, \ldots, \varphi_{2 i-1} \in \mathrm{SAT}$ and $\varphi_{2 i}, \ldots, \varphi_{2 m} \notin \mathrm{SAT}$. In Case 3 in the proof of Claim 4.8 we have seen that there is some minimal upward covering set for $\left(B_{i}, \succ_{i}^{B}\right)$ —call it $M_{i}$ - that corresponds to a satisfying assignment of $\varphi_{2 i-1}$ and that contains all alternatives of $A_{2 i}$. In particular, $M_{i}$ contains $d_{2 i-1}$. For each $j \neq i, 1 \leq j \leq m$, let $M_{j}$ be some minimal upward covering set for $\left(B_{j}, \succ_{j}^{B}\right)$ according to Case 1 (if $\left.j<i\right)$ and Case 2 (if $j>i$ ) in the proof of Claim 4.8 .

In Case 1 we have seen that $d_{2 i-3}$ is upward covered either by $\bar{u}_{1,2 i-3}^{\prime}$ or by $u_{1,2 i-3}^{\prime}$. This is no longer the case, since $d_{2 i-1}$ is in $M_{i}$ and it dominates all alternatives in $A_{2 i-2}$ but not $d_{2 i-3}$. By assumption, $\varphi_{2 i-3}$ is satisfiable, so there exists a minimal upward covering set, which contains $d_{2 i-3}$ as well. Thus, setting

$$
M=\left\{d_{1}, d_{3}, \ldots, d_{2 i-1}\right\} \cup \bigcup_{1 \leq j \leq m} M_{j}
$$

it follows that $M$ is a minimal upward covering set for $(A, \succ)$ containing $d_{1}$.

To show (4.2) from right to left, suppose that $\left\|\left\{i \mid \varphi_{i} \in \mathrm{SAT}\right\}\right\|$ is even. For a contradiction, suppose that there exists some minimal upward covering set $M$ for $(A, \succ)$ that contains $d_{1}$. If $\varphi_{1} \notin \mathrm{SAT}$ then we immediately obtain a contradiction by the argument in the proof of Theorem 4.1. On the other hand, if $\varphi_{1} \in \mathrm{SAT}$ then our assumption that $\left\|\left\{i \mid \varphi_{i} \in \mathrm{SAT}\right\}\right\|$ is even implies that $\varphi_{2} \in \mathrm{SAT}$. It follows from the proof of Claim 4.3 that every minimal upward covering set for $(A, \succ)$ (thus, in particular, $M$ ) contains either $\left\{u_{1,2 i}, u_{1,2 i}^{\prime}\right\}$ or $\left\{\bar{u}_{1,2 i}, \bar{u}_{1,2 i}^{\prime}\right\}$, but not both, and that none of the $e_{j, 2 i}$ and $e_{j, 2 i}^{\prime}$ is in $M$. By the argument presented in Case 3 in the proof of Claim 4.8, the only way to prevent $d_{1}$ from being upward covered by an element of $M$, either $u_{1,2}^{\prime}$ or $\bar{u}_{1,2}^{\prime}$, is to include $d_{3}$ in $M$ as well 6 By applying the same argument $m-1$ times, we will eventually reach a contradiction, since $d_{2 m-1} \in M$ can no longer be prevented from being upward covered by an element of $M$, either $u_{1,2 m}^{\prime}$ or $\bar{u}_{1,2 m}^{\prime}$. Thus, no minimal upward covering set $M$ for $(A, \succ)$ contains $d_{1}$, which completes the proof of (4.2). $\square$

Furthermore, it holds that $\left\|\left\{i \mid \varphi_{i} \in \mathrm{SAT}\right\}\right\|$ is odd if and only if $d_{1}$ is contained in all minimum-size upward covering sets for $A$. This is true since the minimal upward covering sets for $A$ that contain $d_{1}$ are those that correspond to some satisfying assignment for all satisfiable formulas $\varphi_{i}$, and as we have seen in the analysis of Construction 4.2 and the proof sketch of Theorem 4.1, these are the minimum-size upward covering sets for $A$.

\footnotetext{
${ }^{6}$ This implies that $d_{1}$ is not upward covered by either $u_{1,2}^{\prime}$ or $\bar{u}_{1,2}^{\prime}$, since $d_{3}$ dominates them both but not $d_{1}$.
} 


\subsection{Minimal and Minimum-Size Downward Covering Sets}

Turning now to the constructions used to show complexity results about minimal/minimum-size downward covering sets, we will again start by giving a proof sketch of a result due to Brandt and Fischer [BF08], since the following constructions and proofs are based on their construction and proof.

Theorem 4.11 (Brandt and Fischer [BF08]) Deciding whether a designated alternative is contained in some minimal downward covering set for a given dominance graph is NP-hard (i.e., $\mathrm{MC}_{\mathrm{d}}$-MEMBER is NP-hard), even if a downward covering set is guaranteed to exist.

Proof Sketch. NP-hardness of $\mathrm{MC}_{\mathrm{d}}$-MEMBER is again shown by a reduction from SAT. Given a boolean formula in conjunctive normal form, $\varphi\left(v_{1}, v_{2}, \ldots, v_{n}\right)=c_{1} \wedge c_{2} \wedge \cdots \wedge c_{r}$, over the set $V=\left\{v_{1}, v_{2}, \ldots, v_{n}\right\}$ of variables, construct a dominance graph $(A, \succ)$ as follows. The set of alternatives is

$$
A=\left\{x_{i}, \bar{x}_{i}, x_{i}^{\prime}, \bar{x}_{i}^{\prime}, x_{1}^{\prime \prime}, \bar{x}_{i}^{\prime \prime} \mid v_{i} \in V\right\} \cup\left\{y_{j}, z_{j} \mid c_{j} \text { is a clause in } \varphi\right\} \cup\{d\},
$$

where the membership of alternative $d$ in a minimal downward covering set is to be decided. The dominance relation $\succ$ is defined as follows:

- For each $i, 1 \leq i \leq n$, there is a cycle $x_{i} \succ \bar{x}_{i} \succ x_{i}^{\prime} \succ \bar{x}_{i}^{\prime} \succ x_{i}^{\prime \prime} \succ \bar{x}_{i}^{\prime \prime} \succ x_{i}$ with two nested three-cycles, $x_{i} \succ x_{i}^{\prime} \succ x_{i}^{\prime \prime} \succ x_{i}$ and $\bar{x}_{i} \succ \bar{x}_{i}^{\prime} \succ \bar{x}_{i}^{\prime \prime} \succ \bar{x}_{i}$;

- if variable $v_{i}$ occurs in clause $c_{j}$ as a positive literal, then $y_{j} \succ x_{i}$;

- if variable $v_{i}$ occurs in clause $c_{j}$ as a negative literal, then $y_{j} \succ \bar{x}_{i}$;

- for each $j, 1 \leq j \leq r$, we have $d \succ y_{j}$ and $z_{j} \succ d$; and

- for each $i$ and $j$ with $1 \leq i, j \leq r$ and $i \neq j$, we have $z_{i} \succ y_{j}$.

Brandt and Fischer [BF08] showed that there is a minimal downward covering set containing $d$ if and only if $\varphi$ is satisfiable. An example of this reduction is shown in Figure 5 for the boolean formula $\left(v_{1} \vee \neg v_{2} \vee v_{3}\right) \wedge$ $\left(\neg v_{1} \vee \neg v_{3}\right)$. The set $\left\{x_{1}, x_{1}^{\prime}, x_{1}^{\prime \prime}, x_{2}, x_{2}^{\prime}, x_{2}^{\prime \prime}, \bar{x}_{3}, \bar{x}_{3}^{\prime}, \bar{x}_{3}^{\prime \prime}, y_{1}, y_{2}, z_{1}, z_{2}, d\right\}$ is a minimal downward covering set for the dominance graph shown in Figure 5 This set corresponds to the truth assignment that sets $v_{1}$ and $v_{2}$ to true and $v_{3}$ to false, and it contains the designated alternative $d$.

Regarding their construction sketched above, Brandt and Fischer [BF08] showed that every minimal downward covering set for $A$ must contain exactly three alternatives for every variable $v_{i}$ (either $x_{i}, x_{i}^{\prime}$, and $x_{i}^{\prime \prime}$, or $\bar{x}_{i}, \bar{x}_{i}^{\prime}$, and $\left.\bar{x}_{i}^{\prime \prime}\right)$, and the undominated alternatives $z_{1}, \ldots, z_{r}$. Thus, each minimal downward covering set for $A$ consists of at least $3 n+r$ alternatives and induces a truth assignment $\alpha$ for $\varphi$. The number of alternatives contained in any minimal downward covering set for $A$ corresponding to an assignment $\alpha$ is $3 n+r+k$, where $k$ is the number of clauses that are satisfied if $\alpha$ is an assignment not satisfying $\varphi$, and where $k=r+1$ if $\alpha$ is a satisfying assignment for $\varphi$. As a consequence, minimum-size downward covering sets for $A$ correspond to those assignments for $\varphi$ that satisfy the least possible number of clauses of $\varphi \cdot 7$

\footnotetext{
${ }^{7}$ This is different from the case of minimum-size upward covering sets for the dominance graph constructed in the proof sketch of Theorem 4.1 The construction in the proof sketch of Theorem 4.11 cannot be used to obtain complexity results for minimum-size downward covering sets in the same way as the construction in the proof sketch of Theorem 4.1 was used to obtain complexity results for minimum-size upward covering sets.
} 


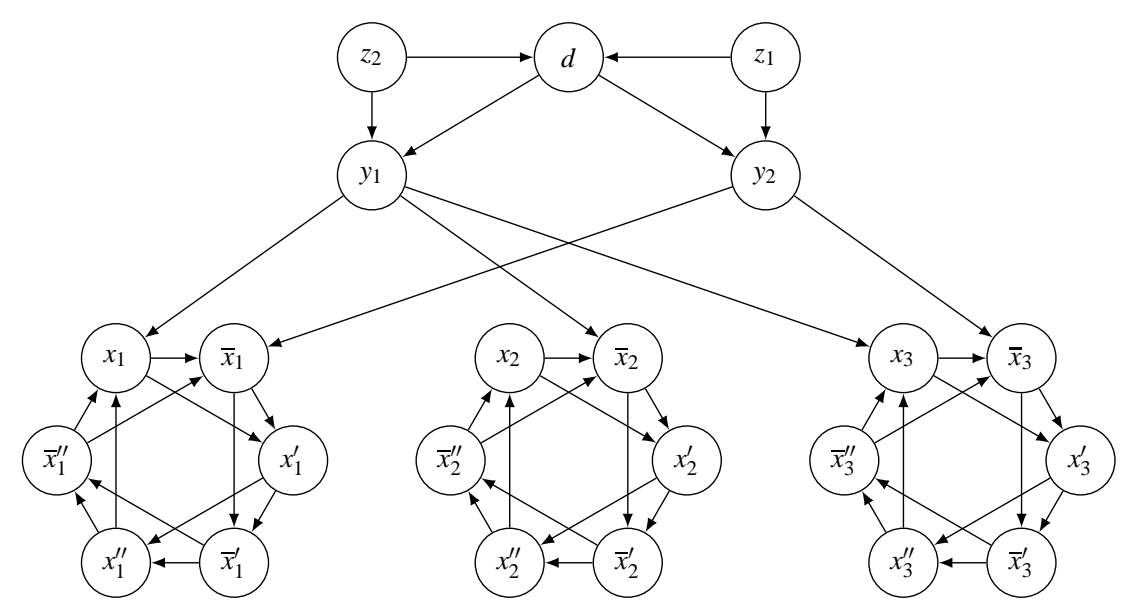

Figure 5: Dominance graph for Theorem 4.11, example for the formula $\left(v_{1} \vee \neg v_{2} \vee v_{3}\right) \wedge\left(\neg v_{1} \vee \neg v_{3}\right)$.

Next, we provide a different construction to transform a given boolean formula into a dominance graph. This construction will later be merged with the construction from the proof sketch of Theorem 4.11 so as to apply Lemma 4.6 to downward covering set problems.

Construction 4.12 (To be used for showing NP- and coNP-hardness for downward covering set problems) Given a boolean formula in conjunctive normal form, $\varphi\left(w_{1}, w_{2}, \ldots, w_{k}\right)=f_{1} \wedge f_{2} \wedge \cdots \wedge f_{\ell}$, over the set $W=\left\{w_{1}, w_{2}, \ldots, w_{k}\right\}$ of variables, we construct a dominance graph $(A, \succ)$. The set of alternatives is

$$
A=A_{1} \cup A_{2} \cup\left\{\widehat{a} \mid a \in A_{1} \cup A_{2}\right\} \cup\{b, c, d\}
$$

with $A_{1}=\left\{x_{i}, x_{i}^{\prime}, x_{i}^{\prime \prime}, \bar{x}_{i}, \bar{x}_{i}^{\prime}, \bar{x}_{i}^{\prime \prime}, z_{i}, z_{i}^{\prime}, z_{i}^{\prime \prime} \mid w_{i} \in W\right\}$ and $A_{2}=\left\{y_{j} \mid f_{j}\right.$ is a clause in $\left.\varphi\right\}$, and the dominance relation $\succ$ is defined by:

- For each $i, 1 \leq i \leq k$, there is, similarly to the construction in the proof of Theorem 4.11 a cycle $x_{i} \succ$ $\bar{x}_{i} \succ x_{i}^{\prime} \succ \bar{x}_{i}^{\prime} \succ x_{i}^{\prime \prime} \succ \bar{x}_{i}^{\prime \prime} \succ x_{i}$ with two nested three-cycles, $x_{i} \succ x_{i}^{\prime} \succ x_{i}^{\prime \prime} \succ x_{i}$ and $\bar{x}_{i} \succ \bar{x}_{i}^{\prime} \succ \bar{x}_{i}^{\prime \prime} \succ \bar{x}_{i}$, and additionally we have $z_{i}^{\prime} \succ z_{i} \succ x_{i}, z_{i}^{\prime \prime} \succ z_{i} \succ \overline{x_{i}}, z_{i}^{\prime} \succ x_{i}, z_{i}^{\prime \prime} \succ \bar{x}_{i}$, and $d \succ z_{i}$;

- if variable $w_{i}$ occurs in clause $f_{j}$ as a positive literal, then $x_{i} \succ y_{j}$;

- if variable $w_{i}$ occurs in clause $f_{j}$ as a negative literal, then $\bar{x}_{i} \succ y_{j}$;

- for each $a \in A_{1} \cup A_{2}$, we have $b \succ \widehat{a}, a \succ \widehat{a}$, and $\widehat{a} \succ d$;

- for each $j, 1 \leq j \leq \ell$, we have $d \succ y_{j}$; and

- $c \succ d$.

An example for this construction is shown in Figure 6 for the boolean formula $\left(\neg w_{1} \vee w_{2} \vee w_{3}\right) \wedge\left(\neg w_{2} \vee\right.$ $\left.\neg w_{3}\right)$, which can be satisfied by setting for example each of $w_{1}, w_{2}$, and $w_{3}$ to false. A minimal downward covering set corresponding to this assignment is $M=\{b, c\} \cup\left\{\bar{x}_{i}, \bar{x}_{i}^{\prime}, \bar{x}_{i}^{\prime \prime}, z_{i}^{\prime}, z_{i}^{\prime \prime} \mid 1 \leq i \leq 3\right\}$. Obviously, the undominated alternatives $b, c, z_{i}^{\prime}$, and $z_{i}^{\prime \prime}, 1 \leq i \leq 3$, are contained in every minimal downward covering set for the dominance graph constructed. The alternative $d$, however, is not contained in any minimal downward 


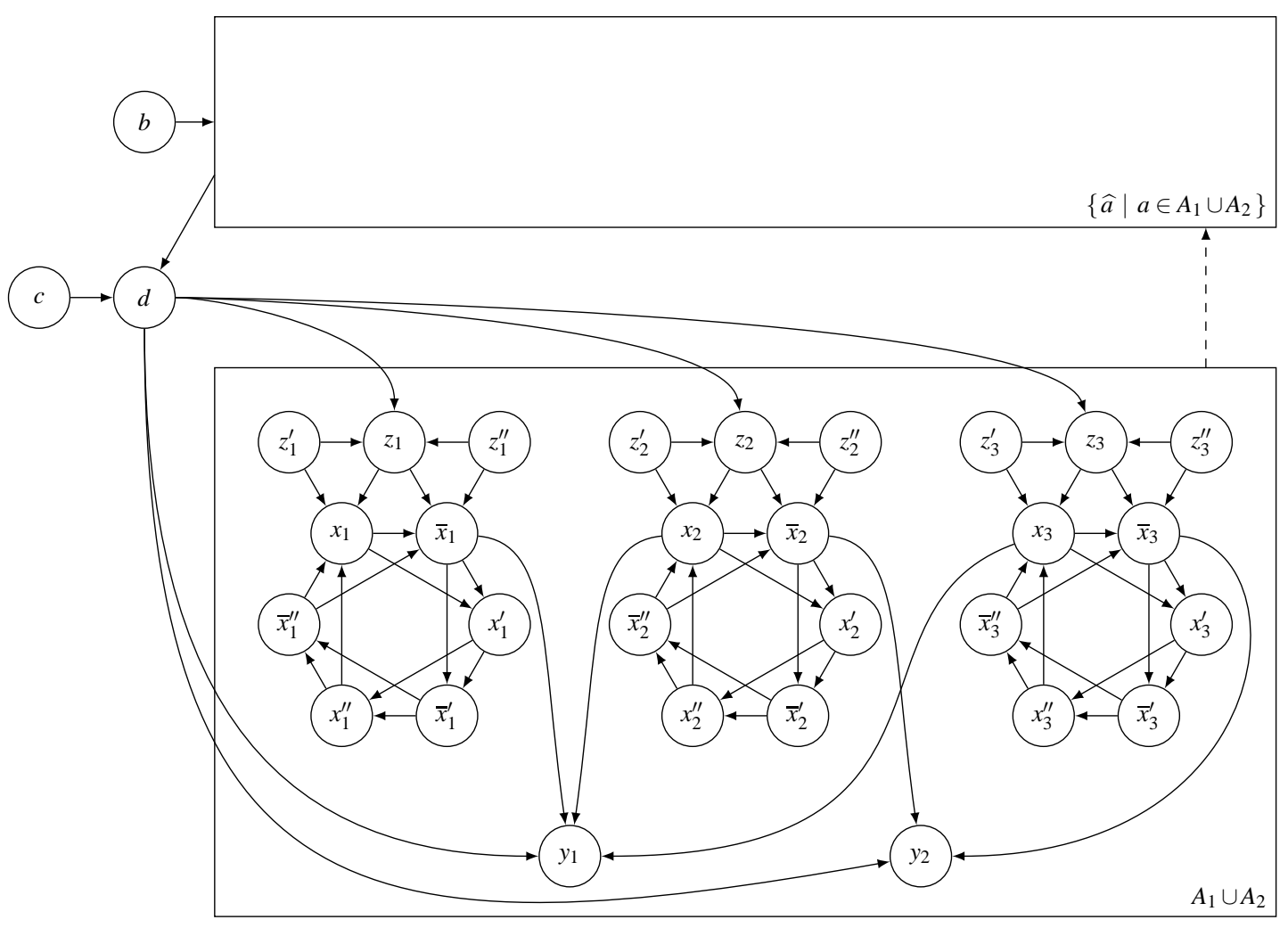

Figure 6: Dominance graph resulting from the formula $\left(\neg w_{1} \vee w_{2} \vee w_{3}\right) \wedge\left(\neg w_{2} \vee \neg w_{3}\right)$ according to Construction 4.12. An edge incident to a set of alternatives represents an edge incident to each alternative in the set. The dashed edge indicates that $a \succ \widehat{a}$ for each $a \in A_{1} \cup A_{2}$.

covering set for $A$. This can be seen as follows. If $d$ were contained in some minimal downward covering set $M^{\prime}$ for $A$ then none of the alternatives $\widehat{a}$ with $a \in A_{1} \cup A_{2}$ would be downward covered. Hence, all alternatives in $A_{1} \cup A_{2}$ would necessarily be in $M^{\prime}$, since they all dominate a different alternative in $M^{\prime}$. But then $M^{\prime}$ is no minimal downward covering set for $A$, since the minimal downward covering set $M$ for $A$ is a strict subset of $M^{\prime}$.

We now show some properties of Construction 4.12 in general.

Claim 4.13 Minimal downward covering sets are guaranteed to exist for the dominance graph defined in Construction 4.12

Proof. The set $A$ of all alternatives is a downward covering set for itself. Hence, there always exists a minimal downward covering set for the dominance graph defined in Construction 4.12

Claim 4.14 Consider the dominance graph $(A, \succ)$ created by Construction 4.12 For each minimal downward covering set $M$ for $A$, if $M$ contains the alternative $d$ then all other alternatives are contained in $M$ as well (i.e., $A=M)$.

Proof. If $d$ is contained in some minimal downward covering set $M$ for $A$, then $\{a, \widehat{a}\} \subseteq M$ for every $a \in$ $A_{1} \cup A_{2}$. To see this, observe that for an arbitrary $a \in A_{1} \cup A_{2}$ there is no $a^{\prime} \in A$ with $a^{\prime} \succ \widehat{a}$ and $a^{\prime} \succ d$ or with $a^{\prime} \succ a$ and $a^{\prime} \succ \widehat{a}$. Since the alternatives $c$ and $b$ are undominated, they are also in $M$, so $M=A$. 
Claim 4.15 Consider Construction 4.12 The boolean formula $\varphi$ is satisfiable if and only if there is no minimal downward covering set for A that contains $d$.

Proof. For the direction from left to right, consider a satisfying assignment $\alpha: W \rightarrow\{0,1\}$ for $\varphi$, and define the set

$$
B_{\alpha}=\{b, c\} \cup\left\{x_{i}, x_{i}^{\prime}, x_{i}^{\prime \prime} \mid \alpha\left(w_{i}\right)=1\right\} \cup\left\{\bar{x}_{i}, \bar{x}_{i}^{\prime}, \bar{x}_{i}^{\prime \prime} \mid \alpha\left(w_{i}\right)=0\right\} \cup\left\{z_{i}^{\prime}, z_{i}^{\prime \prime} \mid 1 \leq i \leq k\right\} .
$$

It is not hard to verify that $B_{\alpha}$ is a minimal downward covering set for $A$. Thus, there exists a minimal downward covering set for $A$ that does not contain $d$. If there were a minimal downward covering set $M$ for $A$ that contains $d$, Claim 4.14 would imply that $M=A$. However, since $B_{\alpha} \subset A=M$, this contradicts minimality, so no minimal downward covering set for $A$ can contain $d$.

For the direction from right to left, assume that no minimal downward covering set for $A$ contains $d$. Since by Claim 4.13 minimal downward covering sets are guaranteed to exist for the dominance graph defined in Construction 4.12, there exists a minimal downward covering set $B$ for $A$ that does not contain $d$, so $B \neq A$. It holds that $\left\{z_{i} \mid w_{i}\right.$ is a variable in $\left.\varphi\right\} \cap B=\emptyset$ and $\left\{y_{j} \mid f_{j}\right.$ is a clause in $\left.\varphi\right\} \cap B=\emptyset$, for otherwise a contradiction would follow by observing that there is no $a \in A$ with $a \succ d$ and $a \succ z_{i}, 1 \leq i \leq k$, or with $a \succ d$ and $a \succ y_{j}$, $1 \leq j \leq \ell$. Furthermore, we have $x_{i} \notin B$ or $\bar{x}_{i} \notin B$, for each variable $w_{i} \in W$. By external stability, for each clause $f_{j}$ there must exist an alternative $a \in B$ with $a \succ y_{j}$. By construction and since $d \notin B$, we must have either $a=x_{i}$ for some variable $w_{i}$ that occurs in $f_{j}$ as a positive literal, or $a=\bar{x}_{i}$ for some variable $w_{i}$ that occurs in $f_{j}$ as a negative literal. Now define $\alpha: W \rightarrow\{0,1\}$ such that $\alpha\left(w_{i}\right)=1$ if $x_{i} \in B$, and $\alpha\left(w_{i}\right)=0$ otherwise. It is readily appreciated that $\alpha$ is a satisfying assignment for $\varphi$.

Claim 4.16 Consider Construction 4.12 The boolean formula $\varphi$ is not satisfiable if and only if there is a unique minimal downward covering set for $A$.

Proof. We again assume that if $\varphi$ is satisfiable, it has at least two satisfying assignments. If $\varphi$ is not satisfiable, there must be a minimal downward covering set for $A$ that contains $d$ by Claim 4.15, and by Claim 4.14 there must be a minimal downward covering set for $A$ containing all alternatives. Hence, there is a unique minimal downward covering set for $A$. Conversely, if there is a unique minimal downward covering set for $A, \varphi$ cannot be satisfiable, since otherwise there would be at least two distinct minimal downward covering sets for $A$, corresponding to the distinct truth assignments for $\varphi$, which would yield a contradiction.

In the dominance graph created by Construction 4.12, the minimal downward covering sets for $A$ coincide with the minimum-size downward covering sets for $A$. If $\varphi$ is not satisfiable, there is only one minimal downward covering set for $A$, so this is also the only minimum-size downward covering set for $A$, and if $\varphi$ is satisfiable, the minimal downward covering sets for $A$ correspond to the satisfying assignments of $\varphi$. As we have seen in the proof of Claim 4.15, these minimal downward covering sets for $A$ always consist of $5 k+2$ alternatives. Thus, they each are also minimum-size downward covering sets for $A$.

Merging the construction from the proof sketch of Theorem 4.11 with Construction 4.12, we will again provide a reduction applying Lemma 4.6, this time to downward covering set problems.

Construction 4.17 (For applying Lemma 4.6 to downward covering set problems) We again apply Wagner's Lemma with the NP-complete problem $S=$ SAT and construct a dominance graph. Fix an arbitrary $m \geq 1$ and let $\varphi_{1}, \varphi_{2}, \ldots, \varphi_{2 m}$ be $2 m$ boolean formulas in conjunctive normal form such that the satisfiability of $\varphi_{j}$ implies the satisfiability of $\varphi_{j-1}$, for each $j \in\{2, \ldots, 2 m\}$. 
We will now define a polynomial-time computable function $f$, which maps the given $2 m$ boolean formulas to a dominance graph $(A, \succ)$ that has useful properties for our downward covering set problems. The set of alternatives is

$$
A=\left(\bigcup_{i=1}^{2 m} A_{i}\right) \cup\left(\bigcup_{i=1}^{m}\left\{r_{i}, s_{i}, t_{i}\right\}\right) \cup\left\{c^{*}, d^{*}\right\}
$$

and the dominance relation $\succ$ on $A$ is defined by

$$
\left(\bigcup_{i=1}^{2 m} \succ_{i}\right) \cup\left(\bigcup_{i=1}^{m}\left\{\left(r_{i}, d_{2 i-1}\right),\left(r_{i}, d_{2 i}\right),\left(s_{i}, r_{i}\right),\left(s_{i}, d_{2 i-1}\right),\left(t_{i}, r_{i}\right),\left(t_{i}, d_{2 i}\right)\right\}\right) \cup\left(\bigcup_{i=1}^{k}\left\{\left(d^{*}, r_{i}\right)\right\}\right) \cup\left\{\left(c^{*}, d^{*}\right)\right\},
$$

where we use the following notation:

1. For each $i, 1 \leq i \leq m$, let $\left(A_{2 i-1}, \succ_{2 i-1}\right)$ be the dominance graph that results from the formula $\varphi_{2 i-1}$ according to Brandt and Fischer's construction given in the proof sketch of Theorem 4.11] We will again use the same names for the alternatives in $A_{2 i-1}$ as in that proof sketch, except that we attach the subscript $2 i-1$.

2. For each $i, 1 \leq i \leq m$, let $\left(A_{2 i}, \succ_{2 i}\right)$ be the dominance graph that results from the formula $\varphi_{2 i}$ according to Construction 4.12. We will again use the same names for the alternatives in $A_{2 i}$ as in that construction, except that we attach the subscript $2 i$.

3. For each $i, 1 \leq i \leq m$, the dominace graphs $\left(A_{2 i-1}, \succ_{2 i-1}\right)$ and $\left(A_{2 i}, \succ_{2 i}\right)$ are connected by the alternatives $s_{i}, t_{i}$, and $r_{i}$ (which play a similar role as the alternatives $z_{i}, z_{i}^{\prime}$, and $z_{i}^{\prime \prime}$ for each variable in Construction 4.12). The resulting dominance graph is denoted by $\left(B_{i}, \succ_{i}^{B}\right)$.

4. Connect the $m$ dominance graphs $\left(B_{i}, \succ_{i}^{B}\right), 1 \leq i \leq m$ (again similarly as in Construction 4.12). The alternative $c^{*}$ dominates $d^{*}$, and $d^{*}$ dominates the $m$ alternatives $r_{i}, 1 \leq i \leq m$.

This construction is illustrated in Figure 7 Clearly, $(A, \succ)$ is computable in polynomial time.

Claim 4.18 Consider Construction 4.17 For each $i, 1 \leq i \leq 2 m$, let $M_{i}$ be the minimal downward covering set for $\left(A_{i}, \succ_{i}\right)$. Then each of the sets $M_{i}$ must be contained in every minimal downward covering set for $(A, \succ)$.

Proof. For each $i, 1 \leq i \leq 2 m$, the only alternative in $A_{i}$ dominated from outside $A_{i}$ is $d_{i}$. Since $d_{i}$ is also dominated by the undominated alternative $z_{1, i} \in A_{i}$ for odd $i$, and by the undominated alternative $c_{i} \in A_{i}$ for even $i$, it is readily appreciated that internal and external stability with respect to elements of $A_{i}$ only depends on the restriction of the dominance graph to $A_{i}$.

Claim 4.19 Consider Construction 4.17 It holds that

$$
\begin{aligned}
& \left\|\left\{i \mid \varphi_{i} \in \mathrm{SAT}\right\}\right\| \text { is odd } \\
& \quad \Longleftrightarrow d^{*} \text { is contained in some minimal downward covering set } M \text { for } A .
\end{aligned}
$$

Proof. For the direction from left to right in (4.3), assume that $\left\|\left\{i \mid \varphi_{i} \in \mathrm{SAT}\right\}\right\|$ is odd. Thus, there is some $j \in\{1, \ldots, m\}$ such that $\varphi_{1}, \varphi_{2}, \ldots, \varphi_{2 j-1}$ are each satisfiable and $\varphi_{2 j}, \varphi_{2 j+1}, \ldots, \varphi_{2 m}$ are each not. Define

$$
M=\left(\bigcup_{i=1}^{2 m} M_{i}\right) \cup\left(\bigcup_{i=1}^{m}\left\{s_{i}, t_{i}\right\}\right) \cup\left\{r_{j}, c^{*}, d^{*}\right\},
$$




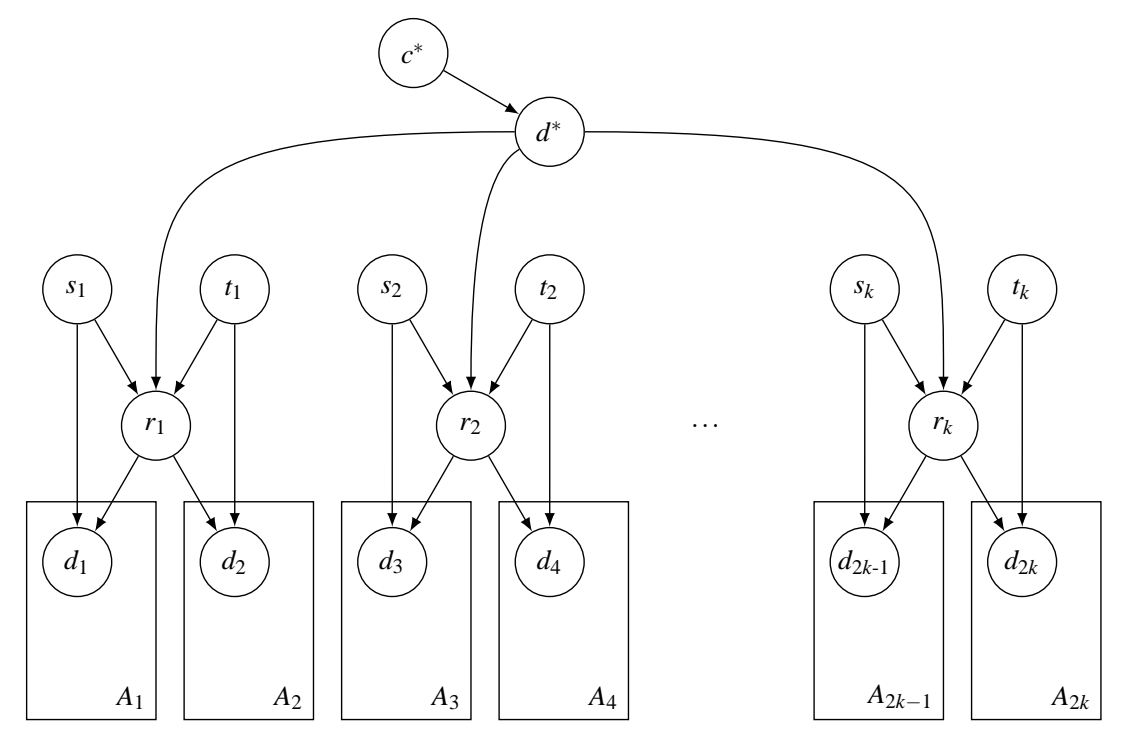

Figure 7: Dominance graph from Construction 4.17

where for each $i, 1 \leq i \leq 2 m, M_{i}$ is some minimal downward covering set of the restriction of the dominance graph to $A_{i}$, satisfying that $d_{i} \in M_{i}$ if and only if

1. $i$ is odd and $\varphi_{i}$ is satisfiable, or

2. $i$ is even and $\varphi_{i}$ is not satisfiable.

Such sets $M_{i}$ exist by the proof sketch of Theorem 4.11 and by Claim 4.15. In particular, $\varphi_{2 j-1}$ is satisfiable and $\varphi_{2 j}$ is not, so $\left\{d_{2 j-1}, d_{2 j}\right\} \subseteq M$. There is no alternative that dominates $d_{2 j-1}, d_{2 j}$, and $r_{j}$. Thus, $r_{j}$ must be in $M$. The other alternatives $r_{i}, 1 \leq i \leq m$ and $i \neq j$, are downward covered by either $s_{i}$ if $d_{2 i-i} \notin M$, or $t_{i}$ if $d_{2 i} \notin M$. Finally, $d^{*}$ cannot be downward covered, because $d^{*} \succ r_{j}$ and no alternative dominates both $d^{*}$ and $r_{j}$. Internal and external stability with respect to the elements of $M_{i}$, as well as minimality of $\bigcup_{i=1}^{2 k} M_{i}$, follow from the proofs of Theorem 4.11 and Claim 4.15. All other elements of $M$ are undominated and thus contained in every downward covering set. We conclude that $M$ is a minimal downward covering set for $A$ that contains $d^{*}$.

For the direction from right to left in (4.3), assume that there exists a minimal downward covering set $M$ for $A$ with $d^{*} \in M$. By internal stability, there must exist some $j, 1 \leq j \leq k$, such that $r_{j} \in M$. Thus, $d_{2 j-1}$ and $d_{2 j}$ must be in $M$, too. It then follows from the proof sketch of Theorem 4.11 and Claim 4.15 that $\varphi_{2 j-1}$ is satisfiable and $\varphi_{2 j}$ is not. Hence, $\left\|\left\{i \mid \varphi_{i} \in \mathrm{SAT}\right\}\right\|$ is odd.

By the remark made after Theorem 4.11, Construction 4.17 cannot be used straightforwardly to obtain complexity results for minimum-size downward covering sets.

\section{Proof of Theorem 3.1}

In this section, we prove Theorem 3.1 by applying the constructions and the properties of the resulting dominance graphs presented in Section 4 . We start with the results on minimal and minimum-size upward covering sets. 


\subsection{Minimal and Minimum-Size Upward Covering Sets}

Theorem 5.1 It is NP-complete to decide, given a dominance graph $(A, \succ)$ and a positive integer $k$, whether there exists a minimal/minimum-size upward covering set for $A$ of size at most $k$. That is, both $\mathrm{MC}_{\mathrm{u}}$-SIZE and $\mathrm{MSC}_{\mathrm{u}}$-SIZE are NP-complete.

Proof. This result can be proven by using the construction of Theorem 4.1 Let $\varphi$ be a given boolean formula in conjunctive normal form, and let $n$ be the number of variables occuring in $\varphi$. Setting the bound $k$ for the size of a minimal/minimum-size upward covering set to $2 n+1$ proves that both problems are hard for NP. Indeed, as we have seen in the paragraph after the proof sketch of Theorem 4.1 there is a size $2 n+1$ minimal upward covering set (and hence a minimum-size upward covering set) for $A$ if and only if $\varphi$ is satisfiable. Both problems are NP-complete, since they can obviously be decided in nondeterministic polynomial time.

Theorem 5.2 Deciding whether a designated alternative is contained in some minimal upward covering set for a given dominance graph is hard for $\Theta_{2}^{p}$ and in $\Sigma_{2}^{p}$. That is, $\mathrm{MC}_{\mathrm{u}}-\mathrm{MEMBER}$ is hard for $\Theta_{2}^{p}$ and in $\Sigma_{2}^{p}$.

Proof. $\quad \Theta_{2}^{p}$-hardness follows directly from Claim 4.10 . For the upper bound, let $(A, \succ)$ be a dominance graph and $d$ a designated alternative in $A$. First, observe that we can verify in polynomial time whether a subset of $A$ is an upward covering set for $A$, simply by checking whether it satisfies internal and external stability. Now, we can guess an upward covering set $B \subseteq A$ with $d \in B$ in nondeterministic polynomial time and verify its minimality by checking that none of its subsets is an upward covering set for $A$. This places the problem in $\mathrm{NP}^{\mathrm{coNP}}$ and consequently in $\Sigma_{2}^{p}$.

Theorem 5.3 1. It is $\Theta_{2}^{p}$-complete to decide whether a designated alternative is contained in some minimum-size upward covering set for a given dominance graph. That is, $\mathrm{MSC}_{\mathrm{u}}$-MEMBER is $\Theta_{2}^{p}$ complete.

2. It is $\Theta_{2}^{p}$-complete to decide whether a designated alternative is contained in all minimum-size upward covering sets for a given dominance graph. That is, $\mathrm{MSC}_{\mathrm{u}}$-MEMBER-ALL is $\Theta_{2}^{p}$-complete.

Proof. By the remark made after Claim 4.10, both problems are hard for $\Theta_{2}^{p}$.

To see that $\mathrm{MSC}_{\mathrm{u}}$-MEMBER is contained in $\Theta_{2}^{p}$, let $(A, \succ)$ be a dominance graph and $d$ a designated alternative in $A$. Obviously, in nondeterministic polynomial time we can decide, given $(A, \succ), x \in A$, and some positive integer $\ell \leq\|A\|$, whether there exists some upward covering set $B$ for $A$ such that $\|B\| \leq \ell$ and $x \in B$. Using this problem as an NP oracle, in $\Theta_{2}^{p}$ we can decide, given $(A, \succ)$ and $d \in A$, whether there exists a minimum-size upward covering set for $A$ containing $d$ as follows. The oracle is asked whether for each pair $(x, \ell)$, where $x \in A$ and $1 \leq \ell \leq\|A\|$, there exists an upward covering set for $A$ of size bounded by $\ell$ that contains the alternative $x$. The number of queries is polynomial (i.e., in $\mathscr{O}\left(\|A\|^{2}\right)$ ), and all queries can be asked in parallel. Having all the answers, determine the size $k$ of a minimum-size upward covering set for $A$, and accept if the oracle answer to $(d, k)$ was yes, otherwise reject.

To show that $\mathrm{MSC}_{\mathrm{u}}$-MEMBER-ALL is in $\Theta_{2}^{p}$, let $(A, \succ)$ be a dominance graph and $d$ a designated alternative in $A$. We now use as our oracle the set of all $((A, \succ), x, \ell)$, where $(A, \succ)$ is our dominance graph, $x \in A$ is an alternative, and $\ell \leq\|A\|$ a positive integer, such that there exists some upward covering set $B$ for $A$ with $\|B\| \leq \ell$ and $x \notin B$. Clearly, this problem is also in NP, and the size $k$ of a minimum-size upward covering set for $A$ can again be determined by asking $\mathscr{O}\left(\|A\|^{2}\right.$ ) queries in parallel (if all oracle answers are no, it holds that $k=\|A\|$ ). 
Now, the $\Theta_{2}^{p}$ machine accepts its input $((A, \succ), d)$ if the oracle answer for the pair $(d, k)$ is no, and otherwise it rejects.

Theorem 5.4 1. (Brandt and Fischer [BF08]) It is coNP-complete to decide whether a designated alternative is contained in all minimal upward covering sets for a given dominance graph. That is, $\mathrm{MC}_{\mathrm{u}}$-MEMBER-ALL is coNP-complete.

2. It is coNP-complete to decide whether a given subset of the alternatives is a minimal upward covering set for a given dominance graph. That is, $\mathrm{MC}_{\mathrm{u}}$-TEST is coNP-complete.

3. It is coNP-hard and in $\Sigma_{2}^{p}$ to decide whether there is a unique minimal upward covering set for a given dominance graph. That is, $\mathrm{MC}_{\mathrm{u}}$-UNIQUE is coNP-hard and in $\Sigma_{2}^{p}$.

Proof. It follows from Claim 4.5 that $\varphi$ is not satisfiable if and only if the entire set of alternatives $A$ is a (unique) minimal upward covering set for $A$. Furthermore, if $\varphi$ is satisfiable, there exists more than one minimal upward covering set for $A$ and none of them contains $e_{1}$ (provided that $\varphi$ has more than one satisfying assignment, which can be ensured, if needed, by adding a dummy variable such that the satisfiability of the formula is not affected). This proves coNP-hardness for all three problems. $\mathrm{MC}_{\mathrm{u}}$-MEMBER-ALL and $\mathrm{MC}_{\mathrm{u}}$-TEST are also contained in coNP, as they can be decided in the positive by checking whether there does not exist an upward covering set that satisfies certain properties related to the problem at hand, so they both are coNP-complete. $\mathrm{MC}_{\mathrm{u}}$-UNIQUE can be decided in the positive by checking whether there exists an upward covering set $M$ such that all sets that are not strict supersets of $M$ are not upward covering sets for the set of all alternatives. Thus, $\mathrm{MC}_{\mathrm{u}}$-UNIQUE is in $\Sigma_{2}^{p}$.

The first statement of Theorem 5.4 was already shown by Brandt and Fischer [BF08]. However, their proof-which uses essentially the reduction from the proof of Theorem 4.1, except that they start from the coNP-complete problem VALIDITY (which asks whether a given formula is valid, i.e., true under every assignment [Pap94]) — does not yield any of the other coNP-hardness results in Theorem 5.4

Theorem 5.5 It is coNP-complete to decide whether a given subset of the alternatives is a minimum-size upward covering set for a given dominance graph. That is, MSC $\mathrm{u}_{\mathrm{u}}$-TEST is coNP-complete.

Proof. This problem is in coNP, since it can be decided in the positive by checking whether the given subset $M$ of alternatives is an upward covering set for the set $A$ of all alternatives (which is easy) and all sets of smaller size than $M$ are not upward covering sets for $A$ (which is a coNP predicate), and coNP-hardness follows directly from Claim 4.5

Theorem 5.6 Deciding whether there exists a unique minimum-size upward covering set for a given dominance graph is hard for coNP and in $\Theta_{2}^{p}$. That is, $\mathrm{MSC}_{\mathrm{u}}$-UNIQUE is coNP-hard and in $\Theta_{2}^{p}$.

Proof. It is easy to see that coNP-hardness follows directly from the coNP-hardness of $\mathrm{MC}_{\mathrm{u}}$-UNIQUE (see Theorem 5.4). Membership in $\Theta_{2}^{p}$ can be proven by using the same oracle as in the proof of the first part of Theorem 5.3. We ask for all pairs $(x, \ell)$, where $x \in A$ and $1 \leq \ell \leq\|A\|$, whether there is an upward covering set $B$ for $A$ such that $\|B\| \leq \ell$ and $x \in B$. Having all the answers, determine the minimum size $k$ of a minimum-size upward covering set for $A$. Accept if there are exactly $k$ distinct alternatives $x_{1}, \ldots, x_{k}$ for which the answer for $\left(x_{i}, k\right), 1 \leq i \leq k$, was yes, otherwise reject. 
An important consequence of the proofs of Theorems 5.4 and 5.6 (and of Construction 4.2 that underpins these proofs) regards the hardness of the search problems $\mathrm{MC}_{\mathrm{u}}$-FIND and $\mathrm{MSC}_{\mathrm{u}}$-FIND.

Theorem 5.7 Assuming $\mathrm{P} \neq \mathrm{NP}$, neither minimal upward covering sets nor minimum-size upward covering sets can be found in polynomial time. That is, neither $\mathrm{MC}_{\mathrm{u}}$-FIND nor $\mathrm{MSC}_{\mathrm{u}}$-FIND are polynomial-time computable unless $\mathrm{P}=\mathrm{NP}$.

Proof. Consider the problem of deciding whether there exists a nontrivial minimal/minimum-size upward covering set, i.e., one that does not contain all alternatives. By Construction 4.2 that is applied in proving Theorems 5.4 and 5.6, there exists a trivial minimal/minimum-size upward covering set for $A$ (i.e., one containing all alternatives in $A$ ) if and only if this set is the only minimal/minimum-size upward covering set for $A$. Thus, the coNP-hardness proof for the problem of deciding whether there is a unique minimal/minimum-size upward covering set for $A$ (see the proofs of Theorem 5.4 and 5.6) immediately implies that the problem of deciding whether there is a nontrivial minimal/minimum-size upward covering set for $A$ is NP-hard. However, since the latter problem can easily be reduced to the search problem (because the search problem, when used as a function oracle, will yield the set of all alternatives if and only if this set is the only minimal/minimumsize upward covering set for $A$ ), it follows that the search problem cannot be solved in polynomial time unless $\mathrm{P}=\mathrm{NP}$.

\subsection{Minimal and Minimum-Size Downward Covering Sets}

Theorem 5.8 It is NP-complete to decide, given a dominance graph $(A, \succ)$ and a positive integer $k$, whether there exists a minimal/minimum-size downward covering set for A of size at most $k$. That is, $\mathrm{MC}_{\mathrm{d}}$-SIZE and $\mathrm{MSC}_{\mathrm{d}}$-SIZE are both NP-complete.

Proof. Membership in NP is obvious, since we can nondeterministically guess a subset $M \subseteq A$ of the alternatives with $\|M\| \leq k$ and can then check in polynomial time whether $M$ is a downward covering set for $A$. NPhardness of $\mathrm{MC}_{\mathrm{d}}-\mathrm{SIZE}$ and $\mathrm{MSC}_{\mathrm{d}}$-SIZE follows from Construction 4.12, the proof of Claim 4.15, and the comments made after Claim 4.16, If $\varphi$ is a given formula with $n$ variables, then there exists a minimal/minimum-size downward covering set of size $5 n+2$ if and only if $\varphi$ is satisfiable.

Theorem 5.9 MSC $_{\mathrm{d}}$-MEMBER, MSC $\mathrm{d}_{\mathrm{d}}$-MEMBER-AlL, and $\mathrm{MSC}_{\mathrm{d}}-\mathrm{UNIQUE}$ are coNP-hard and in $\Theta_{2}^{p}$.

Proof. It follows from Claim 4.16 that $\varphi$ is not satisfiable if and only if the entire set $A$ of all alternatives is the unique minimum-size downward covering set for itself. Moreover, assuming that $\varphi$ has at least two satisfying assignments, if $\varphi$ is satisfiable, there are at least two distinct minimum-size downward covering sets for $A$. This shows that each of $\mathrm{MSC}_{\mathrm{d}}$-MEMBER, MSC $\mathrm{d}_{\mathrm{d}}$-MEMBER-ALL, and $\mathrm{MSC}_{\mathrm{d}}$-UNIQUE is coNP-hard. For all three problems, membership in $\Theta_{2}^{p}$ is shown similarly to the proofs of the corresponding minimum-size upward covering set problems. However, since downward covering sets may fail to exist, the proofs must be slightly adapted. For MSC $\mathrm{d}_{\mathrm{d}}$-MEMBER and $\mathrm{MSC}_{\mathrm{d}}$-UNIQUE, the machine rejects the input if the size $k$ of a mininum-size downward covering set cannot be computed (simply because there doesn't exist any such set). For $\mathrm{MSC}_{\mathrm{d}}$-MEMBER-ALL, if all oracle answers are no, it must be checked whether the set of all alternatives is a downward covering set for itself. If so, the machine accepts the input, otherwise it rejects.

Theorem 5.10 It is coNP-complete to decide whether a given subset is a minimum-size downward covering set for a given dominance graph. That is, $\mathrm{MSC}_{\mathrm{d}}$-TEST is coNP-complete. 
Proof. This problem is in coNP, since its complement (i.e., the problem of deciding whether a given subset of the set $A$ of alternatives is not a minimum-size downward covering set for $A$ ) can be decided in nondeterministic polynomial time. Hardness for coNP follows directly from Claim 4.16.

Theorem 5.11 Deciding whether a designated alternative is contained in some minimal downward covering set for a given dominance graph is hard for $\Theta_{2}^{p}$ and in $\Sigma_{2}^{p}$. That is, $\mathrm{MC}_{\mathrm{d}}-\mathrm{MEMBER}$ is hard for $\Theta_{2}^{p}$ and in $\Sigma_{2}^{p}$.

Proof. Membership in $\Sigma_{2}^{p}$ can be shown analogously to the proof of Theorem 5.2, and $\Theta_{2}^{p}$-hardness follows directly from Claim 4.19

Theorem 5.12 1. (Brandt and Fischer [BF08]) It is coNP-complete to decide whether a designated alternative is contained in all minimal downward covering sets for a given dominance graph. That is, $\mathrm{MC}_{\mathrm{d}}$-MEMBER-AlL is coNP-complete.

2. It is coNP-complete to decide whether a given subset of the alternatives is a minimal downward covering set for a given dominance graph. That is, $\mathrm{MC}_{\mathrm{d}}$-TEST is coNP-complete.

3. It is coNP-hard and in $\Sigma_{2}^{p}$ to decide whether there is a unique minimal downward covering set for a given dominance graph. That is, $\mathrm{MC}_{\mathrm{d}}$-UNIQUE is coNP-hard and in $\Sigma_{2}^{p}$.

Proof. It follows from Claim 4.16 that $\varphi$ is not satisfiable if and only if the entire set of alternatives $A$ is a unique minimal downward covering set for $A$. Furthermore, if $\varphi$ is satisfiable, there exists more than one minimal downward covering set for $A$ and none of them contains $d$ (provided that $\varphi$ has more than one satisfying assignment, which can be ensured, if needed, by adding a dummy variable such that the satisfiability of the formula is not affected). This proves coNP-hardness for all three problems. MC $_{\mathrm{d}}$-MEMBER-ALL and $\mathrm{MC}_{\mathrm{d}}$-TEST are also contained in coNP, because they can be decided in the positive by checking whether there does not exist a downward covering set that satisfies certain properties related to the problem at hand. Thus, they are both coNP-complete. $\mathrm{MC}_{\mathrm{d}}$-UNIQUE can be decided in the positive by checking whether there exists a downward covering set $M$ such that all sets that are not strict supersets of $M$ are not downward covering sets for the set of all alternatives. This shows that $\mathrm{MC}_{\mathrm{d}}$-UNIQUE is in $\Sigma_{2}^{p}$.

The first statement of Theorem 5.12 was already shown by Brandt and Fischer [BF08]. However, their proof-which uses essentially the reduction from the proof of Theorem 4.11, except that they start from the coNP-complete problem VALIDITY - does not yield any of the other coNP-hardness results in Theorem 5.12.

An important consequence of the proofs of Theorems 5.9 and 5.12 regards the hardness of the search problems $\mathrm{MC}_{\mathrm{d}}$-FIND and $\mathrm{MSC}_{\mathrm{d}}$-FIND. (Note that the hardness of $\mathrm{MC}_{\mathrm{d}}$-FIND also follows from a result by Brandt and Fischer [BF08, Thm. 9], see the discussion in Section 3.)

Theorem 5.13 Assuming $\mathrm{P} \neq \mathrm{NP}$, neither minimal downward covering sets nor minimum-size downward covering sets can be found in polynomial time (i.e., neither $\mathrm{MC}_{\mathrm{d}}$-FIND nor $\mathrm{MSC}_{\mathrm{d}}$-FIND are polynomial-time computable unless $\mathrm{P}=\mathrm{NP})$, even when the existence of a downward covering set is guaranteed.

Proof. Consider the problem of deciding whether there exists a nontrivial minimal/minimum-size downward covering set, i.e., one that does not contain all alternatives. By Construction 4.12 that is applied in proving Theorems 5.9 and 5.12, there exists a trivial minimal/minimum-size downward covering set for $A$ (i.e., one 
containing all alternatives in $A$ ) if and only if this set is the only minimal/minimum-size downward covering set for $A$. Thus, the coNP-hardness proof for the problem of deciding whether there is a unique minimal/minimumsize downward covering set for $A$ (see the proofs of Theorems 5.9 and 5.12) immediately implies that the problem of deciding whether there is a nontrivial minimal/minimum-size downward covering set for $A$ is NPhard. However, since the latter problem can easily be reduced to the search problem (because the search problem, when used as a function oracle, will yield the set of all alternatives if and only if this set is the only minimal/minimum-size downward covering set for $A$ ), it follows that the search problem cannot be solved in polynomial time unless $\mathrm{P}=\mathrm{NP}$.

\section{References}

[Alo06] N. Alon. Ranking tournaments. SIAM Journal on Discrete Mathematics, 20(1):137-142, 2006.

[BF08] F. Brandt and F. Fischer. Computing the minimal covering set. Mathematical Social Sciences, 56(2):254-268, 2008.

[BFH07] F. Brandt, F. Fischer, and P. Harrenstein. The computational complexity of choice sets. In Proceedings of the 11th Conference on Theoretical Aspects of Rationality and Knowledge, pages 82-91. ACM Press, 2007.

[BFHM08] F. Brandt, F. Fischer, P. Harrenstein, and M. Mair. A computational analysis of the tournament equilibrium set. In Proceedings of the 23rd AAAI Conference on Artificial Intelligence, pages 3843. AAAI Press, July 2008.

[Bla58] D. Black. The Theory of Committees and Elections. Cambridge University Press, 1958.

[Bor83] G. Bordes. On the possibility of reasonable consistent majoritarian choice: Some positive results. Journal of Economic Theory, 31:122-132, 1983.

[CELM07] Y. Chevaleyre, U. Endriss, J. Lang, and N. Maudet. A short introduction to computational social choice. In Proceedings of the 33rd Conference on Current Trends in Theory and Practice of Computer Science, pages 51-69. Springer-Verlag Lecture Notes in Computer Science \#4362, January 2007.

$\left[\mathrm{CGH}^{+} 88\right]$ J. Cai, T. Gundermann, J. Hartmanis, L. Hemachandra, V. Sewelson, K. Wagner, and G. Wechsung. The boolean hierarchy I: Structural properties. SIAM Journal on Computing, 17(6):1232-1252, 1988.

[CGH $\left.{ }^{+} 89\right]$ J. Cai, T. Gundermann, J. Hartmanis, L. Hemachandra, V. Sewelson, K. Wagner, and G. Wechsung. The boolean hierarchy II: Applications. SIAM Journal on Computing, 18(1):95-111, 1989.

[Con06] V. Conitzer. Computing Slater rankings using similarities among candidates. In Proceedings of the 21st National Conference on Artificial Intelligence, pages 613-619. AAAI Press, July 2006.

[DKNS01] C. Dwork, R. Kumar, M. Naor, and D. Sivakumar. Rank aggregation methods for the web. In Proceedings of the 10th International World Wide Web Conference, pages 613-622. ACM Press, 2001. 
[Dod76] C. Dodgson. A method of taking votes on more than two issues. Pamphlet printed by the Clarendon Press, Oxford, and headed "not yet published" (see the discussions in [MU95, Bla58], both of which reprint this paper), 1876.

[Dut88] B. Dutta. Covering sets and a new Condorcet choice correspondence. Journal of Economic Theory, 44:63-80, 1988.

[FHHR09] P. Faliszewski, E. Hemaspaandra, L. Hemaspaandra, and J. Rothe. A richer understanding of the complexity of election systems. In S. Ravi and S. Shukla, editors, Fundamental Problems in Computing: Essays in Honor of Professor Daniel J. Rosenkrantz, chapter 14, pages 375-406. Springer, 2009.

[Fis77] P. Fishburn. Condorcet social choice functions. SIAM Journal on Applied Mathematics, 33(3):469489, 1977.

[GJ79] M. Garey and D. Johnson. Computers and Intractability: A Guide to the Theory of NPCompleteness. W. H. Freeman and Company, New York, 1979.

[Hem87] L. Hemachandra. The strong exponential hierarchy collapses. In Proceedings of the 19th ACM Symposium on Theory of Computing, pages 110-122. ACM Press, May 1987.

[HHR97a] E. Hemaspaandra, L. Hemaspaandra, and J. Rothe. Exact analysis of Dodgson elections: Lewis Carroll's 1876 voting system is complete for parallel access to NP. Journal of the ACM, 44(6):806$825,1997$.

[HHR97b] E. Hemaspaandra, L. Hemaspaandra, and J. Rothe. Raising NP lower bounds to parallel NP lower bounds. SIGACT News, 28(2):2-13, 1997.

[HHR08] E. Hemaspaandra, L. Hemaspaandra, and J. Rothe. Hybrid elections broaden complexity-theoretic resistance to control. Technical Report arXiv:cs/0608057v2 [cs.GT], ACM Computing Research Repository (CoRR), September 2008. Conference version appeared in Proc. IJCAI'07. Journal version to appear in Mathematical Logic Quarterly, 55(4):397-424, 2009.

[HR98] E. Hemaspaandra and J. Rothe. Recognizing when greed can approximate maximum independent sets is complete for parallel access to NP. Information Processing Letters, 65(3):151-156, 1998.

[HRS06] E. Hemaspaandra, J. Rothe, and H. Spakowski. Recognizing when heuristics can approximate minimum vertex covers is complete for parallel access to NP. R.A.I.R.O. Theoretical Informatics and Applications, 40(1):75-91, 2006.

[HSV05] E. Hemaspaandra, H. Spakowski, and J. Vogel. The complexity of Kemeny elections. Theoretical Computer Science, 349(3):382-391, 2005.

[HW02] E. Hemaspaandra and G. Wechsung. The minimization problem for boolean formulas. SIAM Journal on Computing, 31(6):1948-1958, 2002.

[Kem59] J. Kemeny. Mathematics without numbers. Daedalus, 88:571-591, 1959.

[KSW87] J. Köbler, U. Schöning, and K. Wagner. The difference and truth-table hierarchies for NP. R.A.I.R.O. Informatique théorique et Applications, 21:419-435, 1987. 
[Las97] J. Laslier. Tournament Solutions and Majority Voting. Springer-Verlag, 1997.

[McG53] D. McGarvey. A theorem on the construction of voting paradoxes. Econometrica, 21(4):608-610, 1953.

[Mil80] N. Miller. A new solution set for tournaments and majority voting: Further graph-theoretical approaches to the theory of voting. American Journal of Political Science, 24(1):68-96, 1980.

[MS72] A. Meyer and L. Stockmeyer. The equivalence problem for regular expressions with squaring requires exponential space. In Proceedings of the 13th IEEE Symposium on Switching and Automata Theory, pages 125-129, 1972.

[MU95] I. McLean and A. Urken. Classics of Social Choice. University of Michigan Press, Ann Arbor, Michigan, 1995.

[Pap94] C. Papadimitriou. Computational Complexity. Addison-Wesley, 1994.

[PY84] C. Papadimitriou and M. Yannakakis. The complexity of facets (and some facets of complexity). Journal of Computer and System Sciences, 28(2):244-259, 1984.

[PZ83] C. Papadimitriou and S. Zachos. Two remarks on the power of counting. In Proceedings of the 6th GI Conference on Theoretical Computer Science, pages 269-276. Springer-Verlag Lecture Notes in Computer Science \#145, 1983.

[Rot05] J. Rothe. Complexity Theory and Cryptology. An Introduction to Cryptocomplexity. EATCS Texts in Theoretical Computer Science. Springer-Verlag, Berlin, Heidelberg, New York, 2005.

[RSV03] J. Rothe, H. Spakowski, and J. Vogel. Exact complexity of the winner problem for Young elections. Theory of Computing Systems, 36(4):375-386, 2003.

[Wag87] K. Wagner. More complicated questions about maxima and minima, and some closures of NP. Theoretical Computer Science, 51:53-80, 1987.

[Wag90] K. Wagner. Bounded query classes. SIAM Journal on Computing, 19(5):833-846, 1990.

[Woe03] G. Woeginger. Banks winners in tournaments are difficult to recognize. Social Choice and Welfare, 20:523-528, 2003.

[You77] H. Young. Extending Condorcet's rule. Journal of Economic Theory, 16(2):335-353, 1977. 